\title{
Instability of nondiscrete free subgroups in Lie groups
}

\author{
Alexey GLUTSYUK
}

January 18, 2019

\begin{abstract}
Consider a nondiscrete free subgroup with two generators in a Lie group.

We study the following question stated by Étienne Ghys: is it always possible to make arbitrarily small perturbation of the generators of the free subgroup in such a way that the new group formed by the perturbed generators be not free? In other terms, is it possible to create relations by arbitrarily small perturbation of the generators?

We prove the positive answer.

For certain class (containing the split groups) of real simple Lie groups we study the question (also stated by Ghys) on approximation of free dense subgroups with two generators by nonfree ones. We give an upper bound of the best approximation rate in terms of the length of minimal relation in the approximating nonfree subgroup.
\end{abstract}

We give a survey of related results and open questions.

\section{Contents}

1 Introduction and the plan of the paper $\quad 2$

1.1 Statement of results and open questions . . . . . . . . . . . . . 2

1.2 A simple proof of Theorem 1.1 for $G=P S L_{2}(\mathbb{R}) \ldots \ldots$. . . . . . . 6

1.3 Instability in $c o n j$ - nondegenerate families. The scheme of the proof of Theorem 1.106

1.4 Lengths of relations in approximating subgroups. The scheme of the proof of Theorem 1.13

1.5 Approximations of elements and pairs. Approach to Question 2 . . . . . . . 9

1.6 Historical remarks . . . . . . . . . . . . . . . . . . . . 9

2 Proof of the Main Lemma for weakly split Lie groups 10

2.1 Motivation and proof modulo technical details . . . . . . . . . . . . . . 10

2.2 The iterated commutators $w_{i k}$. The sketch of the proof of Lemma $2.4 \ldots 12$

2.3 Independent eigenvalues. Proof of Lemma $2.7 \ldots \ldots$

3 Case of Lie groups with irreducible adjoint that are not weakly split 18

4 Proof of Theorem 1.1 for arbitrary Lie group 24

$5 \quad$ A short proof of Theorem 1.1 for dense subgroups in $G=P S L_{2}(\mathbb{C}) \quad 26$

6 Proof of Theorem $1.13 \quad 27$

\begin{tabular}{lll}
\hline 7 & References & 31
\end{tabular} 


\section{Introduction and the plan of the paper}

\subsection{Statement of results and open questions}

Let $G$ be a nonsolvable Lie group. It is well-known (see [E]) that almost each (in the sense of the Haar measure) pair of elements $(A, B) \in G \times G$ generates a free subgroup in $G$. At the same time in the case, when $G$ is semisimple, there is a neighborhood $U \subset G \times G$ of unity in $G \times G$ where a topologically-generic pair $(A, B) \in U$ generates a dense subgroup: the latter pairs form an open dense subset in $U$.

The pairs generating groups with relations form a countable union of surfaces (relation surfaces) in $G \times G$. We show that the relation surfaces are dense in $U$.

The main result of the paper is the following

1.1 Theorem Any nondiscrete free subgroup with two generators in a nonsolvable Lie group $G$ is unstable. More precisely, consider two elements $A, B \in G$ generating a free subgroup $\Gamma=<A, B>$. Let $\Gamma$ be not discrete. Then there exists a sequence $\left(A_{k}, B_{k}\right) \rightarrow(A, B)$ of pairs converging to $(A, B)$ such that the corresponding groups $<A_{k}, B_{k}>$ have relations: there exists a sequence $w_{k}=w_{k}(a, b)$ of nontrivial abstract words in symbols $a, b$ and their inverses $a^{-1}, b^{-1}$ such that $w_{k}\left(A_{k}, B_{k}\right)=1$ for all $k$.

1.2 Remark The condition that the subgroup under consideration be nondiscrete is natural: one can provide examples of discrete free subgroups of $P S L_{2}(\mathbb{C})$ (e.g., the Schottky group, see $[\mathrm{AGK}])$ that are stably free, i.e., remain free under any small perturbation of the generators.

The question of instability of nondiscrete free subgroups was stated by É Ghys.

1.3 Remark In the case, when the Lie group under consideration is $P S L_{2}(\mathbb{R})$, Theorem 1.1 easily follows from the density of elliptic elements of finite orders in an open domain of $P S L_{2}(\mathbb{R})$ : the proof is given in Subsection 1.2. A similar argument proves Theorem 1.1 in the case of essentially compact Lie group (i.e., whose adjoint has an invariant positive-definite scalar product). The case of $P S L_{2}(\mathbb{C})$ is already nontrivial (in some sense, this is a first nontrivial case). In this case the previous argument cannot be applied, since elliptic elements in $P S L_{2}(\mathbb{C})$ are nowhere dense. At the same time, there is a short proof of Theorem 1.1 for dense subgroups in $P S L_{2}(\mathbb{C})$ that uses holomorphic motions and quasiconformal mappings. We present it in Section 5 .

Theorem 1.1 is proved in Sections 2-4. Firstly we prove it (in 1.3 and Section 2) for so-called weakly split groups (see Definition 1.12 below) - a class of Lie groups containing all groups $S L_{n}(\mathbb{R})$ and more generally, all the split groups (see [VO]). A reader can read the proofs there assuming everywhere that $G=S L_{n}(\mathbb{R})$.

Then in Section 3 we prove it for all the Lie groups with irreducible adjoint. Afterwards in Section 4 we deduce the statement of Theorem 1.1 for arbitrary Lie group, using the classical decomposition theorems for Lie algebras [VO].

Let us recall what is the adjoint action (see [VO]). The group $G$ acts on itself by conjugations (the unity is fixed). The derivative of this action along the vectors of the tangent Lie algebra $T_{1} G$ defines a linear representation of $G$ in $T_{1} G$ called the adjoint representation. The adjoint representation of an element $g \in G$ is denoted $A d_{g}$, see [VO]. (If $G$ is a matrix group, then the adjoint action is given by matrix conjugation: $A d_{g}(h)=g h g^{-1}$.) 
1.4 Example Let $G=S L_{n}(\mathbb{R})$. The adjoint action of a diagonal matrix $A=\operatorname{diag}\left(a_{1}, \ldots, a_{n}\right)$ is diagonalizable and has the eigenvalues $1, \lambda_{i j}=\frac{a_{i}}{a_{j}}, i \neq j$. The $\lambda_{i j}$ 's correspond to the unipotent matrices with zeros everywhere except for the $(i, j)$ - th element. The other (unit) eigenvalues correspond to the diagonal matrices.

In the case of Lie group with irreducible adjoint we prove the next more precise Theorem. To state it, let us recall the following definition, which is equivalent to the classical one from [VO].

1.5 Definition A Lie group is said to be simple if the adjoint representation of its unity component is irreducible. A Lie group is said to be semisimple, if its unity component has no normal solvable Lie subgroup of positive dimension.

1.6 Remark A disconnected Lie group with irreducible adjoint is always semisimple, but not always simple. For example, take a connected simple Lie group $G^{\prime}$ and put $G$ to be the subgroup of $\operatorname{Aut}\left(G^{\prime} \times G^{\prime}\right)$ generated by the interior automorphisms (i.e. conjugations) and the transposition $(a, b) \mapsto(b, a)$. The group $G$ thus obtained is a lie group that is a semidirect product of $\left(G^{\prime} / C\left(G^{\prime}\right)\right) \times\left(G^{\prime} / C\left(G^{\prime}\right)\right)\left(C\left(G^{\prime}\right)\right.$ is the center of $\left.G^{\prime}\right)$ and the group $\mathbb{Z}_{2}=\{0,1\}$. This is a semisimple group with the unity component $\left(G^{\prime} / C\left(G^{\prime}\right)\right) \times\left(G^{\prime} / C\left(G^{\prime}\right)\right)$. It is not simple: the adjoint of the unity component is reducible, since it leaves invariant the Lie algebras of the left and right groups $G^{\prime}$ (the latter algebras are the only invariant subspaces of the adjoint of the unity component). On the other hand, the adjoint of the disconnected group $G$ is irreducible. Indeed, the previous subalgebras are the only candidates to be invariant subspaces. The adjoint is generated by that of the unity component and by the adjoint of the transposition $T:(x, y) \mapsto(y, x)$. The latter adjoint acts on $T_{I d} A u t\left(G^{\prime} \times G^{\prime}\right)$, which is canonically identified with $T_{1} G^{\prime} \times T_{1} G^{\prime}$. It follows from definition that $A d_{T}$ acts by the transposition of the $T_{1} G^{\prime}$ - components. Thus, neither left, nor right $T_{1} G^{\prime}$ can be $A d_{G^{-}}$ invariant. This together with the previous discussion proves that $A d_{G}$ is irreducible.

1.7 Definition Let $G$ be a Lie group, $\alpha=\left(a_{1}, \ldots, a_{M}\right) \in G^{M}$. Consider the $G$ - action on $G^{M}$ by simultaneous conjugations and denote $\operatorname{Conj}\left(a_{1}, \ldots, a_{M}\right) \subset G^{M}$ the orbit of $\left(a_{1}, \ldots, a_{M}\right)$ (i.e., the joint conjugacy class).

The following Proposition is obvious and well-known.

1.8 Proposition Let $G$ be a semisimple Lie group, $n=\operatorname{dim} G$. Let a pair (or $M$ - ple) of its elements generate a dense subgroup in $G$. Then their joint conjugacy class is the quotient of $G$ by its center. The conjugacy classes corresponding to all the previous pairs $(M-$ ples) form an analytic manifold of dimension $n$ (respectively, $(M-1) n)$. The mapping $\left(a_{1}, \ldots, a_{M}\right) \mapsto \operatorname{Conj}\left(a_{1}, \ldots, a_{M}\right)$ is a local submersion at points $\left(a_{1}, \ldots, a_{M}\right) \in G^{M}$ such that $a_{i}$ generate a dense subgroup.

1.9 Definition Let $G$ be a semisimple Lie group, $\alpha(u)=\left(a_{1}(u), \ldots, a_{M}(u)\right)$ be a family of $M$ - ples of its elements depending on a parameter $u \in \mathbb{R}^{l}$. We say that $\alpha$ is conjnondegenerate at $u=u_{0}$ if the corresponding mapping $u \mapsto \operatorname{Conj}(\alpha(u))$ has a rank no less than $n=\operatorname{dim} G$ at $u=u_{0}$. Otherwise, if the previous rank is less than $n$, we say that the family $\alpha(u)$ is conj- degenerate at $u_{0}$. 
1.10 Theorem Let $G$ be a Lie group with irreducible $A d_{G}$ (not necessarily connected). Consider a family $\alpha(u)=\left(a_{1}(u), \ldots, a_{M}(u)\right), M \in \mathbb{N}$, of $M$-ples of its elements that depend on a parameter $u=\left(u_{1}, \ldots, u_{l}\right) \in \mathbb{R}^{l}$. Suppose that the subgroup $<\alpha(0)>\subset G$ generated by $\alpha(0)$ is dense and for each $u$ the mappings $a_{i}(0) \mapsto a_{i}(u)$ induce a group isomorphism $\langle\alpha(0)>\rightarrow\langle\alpha(u)>$. Then the family $\alpha(u)$ is conj-degenerate at 0 (see the previous Definition).

Theorem 1.10 will be proved in the next two Sections. Firstly we prove it (in Section 2) for the so-called weakly split groups (see Definition 1.12 below). Then in Section 3 we prove it for other Lie groups with irreducible adjoint. In both cases the method of the proof is the same, but it is slightly more technical in the latter case.

Proposition 1.8 and Theorem 1.10 imply immediately Theorem 1.1 in the case, when $A d_{G}$ is irreducible and $A, B$ generate a dense subgroup. Indeed, suppose the contrary: each pair $(a, b)$ close to $(A, B)$ generates a free subgroup, hence, the mapping $(A, B) \mapsto(a, b)$ induces an isomorphism of the corresponding subgroups. Consider the family of all the pairs $(a, b)$ depending on the parameters in $G$ of the elements $a$ and $b$. By the previous assumption, the latter family satisfies the condition of Theorem 1.10, which implies its conj- degeneracy. On the other hand, it is conj- nondegenerate (Proposition [1.8), - a contradiction. Thus, the group $<A, B\rangle$ is not stably free.

Ghys has also proposed to study approximations of free subgroups by nonfree ones in the following sense. It is well-known that for any $\varepsilon>0$ and almost each (more precisely, $\varepsilon$ Diophantine) irrational number $r$ there exists a $C=C(r)>0$ such that for any irreducible fraction $\frac{m}{n} \in \mathbb{Q}$ one has $\left|r-\frac{m}{n}\right|>\frac{C}{n^{2+\varepsilon}}$. This approximation accuracy is optimal in some sense: the continuous fractions give approximations of accuracy no worse than $\frac{1}{n^{2}}$.

Les us say that a pair of elements of a Lie group is irrational, if it generates a free dense subgroup. A pair of elements generating a group with relations will be called rational; its denominator is the minimal length of relation.

Question 1 (É.Ghys). Given a generic irrational pair of elements in a Lie group. What is the optimal asymptotic accuracy of its approximations by rational pairs, as their denominators tend to infinity?

1.11 Remark The number of (reduced) words $w(a, b)$ of a given length $l$ grows exponentially in $l$. This motivates the following

Question 2. Let $G$ be a semisimple Lie group. Is it true that for any irrational pair $(A, B) \in G \times G$ there exist a $c=c(A, B)>0$ and a sequence of rational pairs $\left(A_{r}, B_{r}\right)$ with denominators $l_{r}$ such that

$$
\left(A_{r}, B_{r}\right) \rightarrow(A, B), d_{r}=\operatorname{dist}\left(\left(A_{r}, B_{r}\right),(A, B)\right)<e^{-c l_{r}} \text { for all } r ?
$$

Question 3. In the previous Question is it true that for almost each irrational pair $(A, B)$ the latter approximation rate is optimal: there is a $c_{1}=c_{1}(A, B)>0$ such that for any rational pair $\left(A^{\prime}, B^{\prime}\right)$ with the denominator $l$ one has $\operatorname{dist}\left(\left(A^{\prime}, B^{\prime}\right),(A, B)\right)>e^{-c_{1} l}$ ?

In Subsection 1.4 and Section 6 we prove the next Theorem related to Question 1. To state it, let us introduce the following Definition. 
1.12 Definition A Lie group $G$ is said to be weakly split, if its adjoint is irreducible and there is a domain $U \subset G$ whose closure contains 1 such that the adjoint $A d_{g}$ of each element $g \in U$ satisfies the following conditions:

1) it has a real simple eigenvalue $\lambda>1$;

2) $|\lambda-1|>\left|\lambda^{\prime}-1\right|$ for any other (real or complex) eigenvalue $\lambda^{\prime}$.

1.13 Theorem Let $G$ be a weakly split Lie group. Then for any irrational pair $(A, B) \in$ $G \times G$ there exist a $c=c(A, B)>0$ and a sequence of rational pairs $\left(A_{k}, B_{k}\right) \rightarrow(A, B)$ with denominators $l_{k}$ such that

$$
d_{k}=\operatorname{dist}\left(\left(A_{k}, B_{k}\right),(A, B)\right)<e^{-c \ln ^{2} l_{k}} \text { for all } k .
$$

1.14 Example Consider a group $G=S L_{n}(\mathbb{R})$ and its element that is a real-diagonalizable matrix with distinct eigenvalues such that the pair ratios of the eigenvalues are distinct. Then its adjoint satisfies 1) and 2) (see Example 1.4). Therefore, $G$ is weakly split (with $U$ containing all the previous diagonalizable matrices). More generally, all the split semisimple Lie groups (see $[\mathrm{VO}]$ ) are weakly split.

Counterexamples. Complex semisimple Lie groups (considered as real ones) are obviously not weakly split. The groups $S O(1, n)$ with $n \geq 3$ are also not weakly split, and in general, do not admit complex structure (a remark due to J.-F.Quint).

A question (close to Question 1) concerning Diophantine properties of and individual pair $A, B \in S O(3)$ was studied in [KR]. We say that a pair $(A, B) \in S O(3) \times S O(3)$ is Diophantine (see $[\mathrm{KR}]$ ), if there exists a constant $D>1$ depending on $A$ and $B$ such that for any (reduced) word $w_{k}=w_{k}(a, b)$ of length $k$

$$
\left|w_{k}(A, B)-1\right|>D^{-k}
$$

A.Gamburd, D.Jakobson and P.Sarnak have stated the following

Question 4 [GJS]. Is it true that almost each pair $(A, B) \in S O(3) \times S O(3)$ is Diophantine?

V.Kaloshin and I.Rodnianski $[\mathrm{KR}]$ proved that almost each pair $(A, B)$ satisfies a weaker inequality with the latter right-hand side replaced by $D^{-k^{2}}$.

The following inverse question is closely related to Question 2.

Question 5. Let $G$ be a semisimple Lie group (e.g., $S L_{n}(\mathbb{R})$ ). Is it true that for any $g \in G$ and any irrational pair $A, B \in G$ there exist a $c=c(A, B, g)>0$ and a sequence $\omega_{r}(a, b)$ of words in $a^{ \pm 1}$ and $b^{ \pm 1}$ (denote $l_{r}=\left|\omega_{r}\right|$ their lengths) such that

$$
\omega_{r}(A, B) \rightarrow g, \text { as } r \rightarrow \infty, \text { and } d_{r}=\operatorname{dist}\left(\omega_{r}(A, B), g\right)<e^{-c l_{r}} ?
$$

Question 5 is closely related to Question 2, as is explained in 1.4.

Question 6. Is there an analogue of Theorem 1.1 for the group of

- germs of one-dimensional real diffeomorphisms?

- germs of one-dimensional conformal diffeomorphisms?

- diffeomorphisms of compact manifold? 
The latter question concerning conformal germs is related to study of one-dimensional holomorphic foliations on $\mathbb{C P}^{2}$ with isolated singularities. A generic vector field on $\mathbb{C}^{2}$ with components of degree $n-1$ defines a singular holomorphic foliation on $\mathbb{C P}^{2}$ with invariant line $L$ at infinity; the line $L$ contains $n$ singularities $a_{1}, \ldots, a_{n} \in L$.

Let $\Delta \subset \mathbb{C P}^{2}$ be a transversal cross-section to $L, a$ be its point of intersection with $L$. A circuit in $L$ around each $a_{i}$ with base point at $a$ defines a germ of conformal holonomy mapping $h_{i}:(\Delta, a) \rightarrow(\Delta, a)$. Let us choose the circuit around each $a_{i}$ so that the $n$ circuits generate the fundamental group of $L \backslash\left\{a_{1}, \ldots, a_{n}\right\}$. The monodromy group is the group generated by the holonomy germs $h_{i}$.

Yu.S.Ilyashenko and A.S.Pyartli have proved [IP] that for a generic polynomial vector field of degree greater than two the monodromy group is free. "Generic" means "lying outside at most a countable union of analytic surfaces".

Question 7. Does there exist an open (or open and dense) subset $U$ in the space of polynomial vector fields of fixed degree (say, greater than two) such that the monodromy group of each vector field from $U$ is free?

\subsection{A simple proof of Theorem 1.1 for $G=P S L_{2}(\mathbb{R})$}

Without loss of generality we assume that $\overline{\langle A, B\rangle}=G$. Otherwise, $\langle A, B\rangle$ would be dense in a Lie subgroup of dimension at most two, which is solvable, hence, $A$ and $B$ cannot generate a free subgroup.

There is an open set $U \subset G$ formed by nontrivial elliptic transformations $D_{1} \rightarrow D_{1}$ of the unit disc, which are conformally conjugated to nontrivial rotations. The rotation number (which is the rotation angle divided by $2 \pi$ ) is a local (nowhere zero) analytic function in the parameters of $U$. An elliptic transformation $f$ has finite order if and only if its rotational number $\rho(f)$ is rational.

Let $w=w(a, b)$ be a word such that $w(A, B) \in U$ (it exists by density). It suffices to show that the function $(a, b) \mapsto \rho(w(a, b))$ is not constant near $(A, B)$ : then it follows that there exists a sequence $\left(a_{n}, b_{n}\right) \rightarrow(A, B)$ such that $w\left(a_{n}, b_{n}\right)$ are finite order elements, thus, one has relations of the type $w^{k_{n}}\left(a_{n}, b_{n}\right)=1$.

The previous function is locally analytic. Suppose the contrary: it is constant. Then by analyticity, it is constant globally and $w(a, b)$ is elliptic with one and the same nonzero rotation number for all the pairs $(a, b)$. On the other hand, it vanishes at $(a, b)=(1,1)$, since $w(1,1)=1$ - a contradiction. This proves Theorem 1.1 for $G=P S L_{2}(\mathbb{R})$.

\subsection{Instability in conj- nondegenerate families. The scheme of the proof of Theorem 1.10}

Let $G$ be a Lie group with irreducible $A d_{G}, n=\operatorname{dim} G, \alpha(u)=\left(a_{1}(u), \ldots, a_{M}(u)\right)$ be an $M$ ple family depending smoothly on a parameter $u \in \mathbb{R}^{l}$ and $\alpha(0)$ generate a dense subgroup. We prove Theorem 1.10 by contradiction. Namely, we suppose that $\alpha(u)$ is conj-nondegenerate at 0 , i.e., the mapping $u \mapsto \operatorname{Conj}(\alpha(u))$ has the rank at least $n$ at 0 . Without loss of generality we assume that the parameter $u$ has dimension $n$ and the previous rank equals $n$ (one can achieve this by passing to a subfamily).

Let us show that for appropriate sequence $u_{k} \rightarrow 0$ for each $k$ the subgroup $\left\langle\alpha\left(u_{k}\right)\right\rangle$ has an additional relation between the $a_{i}$ ' $\mathrm{s}$ that does not hold in $\langle\alpha(u)>$ identically in $u$. 
This makes impossible the condition of Theorem 1.10 saying that the mappings $\alpha(0) \mapsto \alpha(u)$ induce isomorphisms $\langle\alpha(0)>\rightarrow<\alpha(u)>$.

Let us motivate the construction. Our goal is to construct a sequence $w_{k}$ of words such that there is a sequence $u_{k} \rightarrow 0$ that solve the equations $w_{k}\left(u_{k}\right)=1$. To guarantee this, it suffices to achieve that $w_{k}(\alpha(0)) \rightarrow 1$ and the function $W_{k}: u \mapsto w_{k}(\alpha(u))$ sends a $\varepsilon_{k^{-}}$ neighborhood of 0 onto a domain containing a $\Delta$ - neighborhood of $W_{k}(0)$, where $\varepsilon_{k} \rightarrow 0$ and $\Delta$ is independent on $k$. Then the latter neighborhood contains 1 , whenever $k$ is large enough, hence, the above solution $u_{k}$ exists in the former $\varepsilon_{k^{-}}$neighborhood of 0 . We do our construction in such a way that $\varepsilon_{k}=O\left(\frac{1}{k}\right)$ and the derivative of the function $W_{k}$ at 0 grows linearly in $k$.

For any $\delta>0$ and $Q=\left(Q_{1}, \ldots, Q_{n}\right) \in \mathbb{R}^{n}$ denote $I_{\delta}(Q)$ the $\delta$ - cube centered at $Q$. Denote $I_{\delta}=I_{\delta}(0)$.

1.15 Lemma (Main Lemma). Let $G, u, \alpha(u)$ be as at the beginning of the Subsection. Then for any element $g \in G$ there exist $c=c(A, B, g), \delta=\delta(A, B, g)>0$ and a sequence $w_{k}\left(a_{1}, \ldots a_{M}\right)$ of words in $M$ elements and their inverses such that

1) the restriction of each mapping $W_{k}: u \mapsto w_{k}(\alpha(u)) \in G$ to the cube $I_{\varepsilon_{k}}, \varepsilon_{k}=\frac{\delta}{k}$, is a composition of the homothety $u \mapsto k u$ (which maps $I_{\varepsilon_{k}}$ onto $I_{\delta}$ ) and a mapping $\phi_{k}: I_{\delta} \rightarrow$ $G$ such that the sequence $\phi_{k}$ (or some its subsequence, if $G$ is not weakly split) converges uniformly with derivatives to a diffeomorphism $\phi: I_{\delta} \rightarrow \phi\left(I_{\delta}\right) \subset G$;

2) the image $\phi\left(I_{\delta}\right)$ contains $g$;

3) the length $l_{k}=\left|w_{k}\right|$ of each word $w_{k}$ is less than $e^{c k}$.

Addendum to Lemma 1.15. Let in the Main Lemma $G$ be weakly split, $g \in G, w_{k}$ be a word sequence from the Lemma. Then one can achieve that in addition there exist a $0<S=S(\alpha, g)<1$ such that

$$
\operatorname{dist}\left(w_{k}(\alpha(0)), g\right)<S^{k} \text { for all } k \text { large enough. }
$$

The Addendum will be used in the proof of Theorem 1.13. The Lemma will be proved in Sections 2 and 3. The Addendum will be proved in Section 2.

Proof of Theorem 1.10 modulo the Main Lemma. Let $g=1, w_{k}, \delta, \varepsilon_{k}=\frac{\delta}{k}, \phi_{k}$, $\phi$ be as in the previous Lemma. Then the image $W_{k}\left(I_{\varepsilon_{k}}\right)$ converges to $\phi\left(I_{\delta}\right)$ (may be after passing to a subsequence) in the Hausdorff topology (statement 1) of the Lemma), and hence, contains 1 whenever $k$ is large enough, as does the latter (statement 2)). This implies that $\phi_{k}\left(\widetilde{u}_{k}\right)=1$ for some $\widetilde{u}_{k}=O\left(\operatorname{dist}\left(w_{k}(\alpha(0)), 1\right)\right)$, and hence,

$$
w_{k}\left(\alpha\left(u_{k}\right)\right)=1, u_{k}=k^{-1} \widetilde{u}_{k}=O\left(\operatorname{dist}\left(w_{k}(\alpha(0)), 1\right)\right) \rightarrow 0, \text { as } k \rightarrow \infty .
$$

This proves Theorem 1.10

\subsection{Lengths of relations in approximating subgroups. The scheme of the proof of Theorem 1.13}

Let $G$ be a weakly split Lie group, $(A, B) \in G \times G$ be an irrational pair. Let $\alpha(u)=(a(u), b(u))$ be its conj- nondegenerate deformation depending on $u \in \mathbb{R}^{n},(a(0), b(0))=(A, B)$. We show 
that there exists a sequence $u_{k} \rightarrow 0$ of the parameter values such that the pairs $\left(A_{k}, B_{k}\right)=$ $\left(a\left(u_{k}\right), b\left(u_{k}\right)\right)$ are rational and satisfy Theorem 1.13 To do this, it suffices to show that there exist a $c^{\prime}=c^{\prime}(A, B)>0$, a sequence $\omega_{k}(a, b)$ of words (denote $l_{k}=\left|\omega_{k}\right|$ ) and a sequence $u_{k} \rightarrow 0$ such that

$$
\omega_{k}\left(a\left(u_{k}\right), b\left(u_{k}\right)\right)=1,\left|u_{k}\right|<e^{-c^{\prime} \ln ^{2} l_{k}} .
$$

We construct $\omega_{k}$ and $u_{k}$ so that there exist $c_{1}, c_{2}>0$ such that

$$
\omega_{k}\left(a\left(u_{k}\right), b\left(u_{k}\right)\right)=1, l_{k}<e^{c_{1} k},\left|u_{k}\right|<e^{-c_{2} k^{2}} \text {, for all } k .
$$

This will imply that (1.5) holds with $c^{\prime}=c_{2} c_{1}^{-2}$.

The Main Lemma (applied to $g=1$ ) implies the existence of sequences of words $w_{k}$ and parameter values $u_{k}, w_{k}\left(a\left(u_{k}\right), b\left(u_{k}\right)\right)=1$, satisfying (1.4) with $l_{k}<e^{c k}, c$ is independent on $k$. If in addition we had

$$
\operatorname{dist}\left(w_{k}(a(0), b(0)), 1\right)<e^{-c_{3} k^{2}},
$$

this together with (1.4) would imply (1.6). On the other hand, the Addendum to the Lemma implies the weaker inequality

$$
\operatorname{dist}\left(w_{k}(a(0), b(0)), 1\right)<e^{-c_{3} k} \text { with } c_{3}>0 \text { independent on } k .
$$

We construct appropriate auxiliary words $\omega_{k k}$ (and put $\omega_{k}=w_{k} \omega_{k k}$ ), $\left|\omega_{k k}\right|=e^{O(k)}$, so that there exists a $c_{3}>0$ such that the new words $\omega_{k}$ satisfy (1.7). Then we deduce (1.6) for the new words $\omega_{k}$. More precisely, we construct (inductively in $j$ in Section 6) auxiliary words $\omega_{j k}, j=2, \ldots, k$, so that there exist $c_{5}, c_{6}, c_{7}, \delta^{\prime}>0$ (independent on $j$ and $k$ ) such that

$$
\begin{gathered}
\operatorname{dist}\left(\omega_{j k}(A, B), w_{k}^{-1}(A, B)\right)<e^{-c_{5} j k}, \\
l_{j k}=\left|\omega_{j k}\right|<e^{c_{6} k},
\end{gathered}
$$

the derivative of each mapping $u \mapsto \omega_{j k}(a(u), b(u))$ has norm less than $c_{7}$ in the cube $I_{\delta^{\prime}}$.

Then the word $\omega_{k k}$ is a one we are looking for. Indeed, it suffices to show that each equation $w_{k} \omega_{j k}(a(u), b(u))=1$ has a solution

$$
u_{j k}=O\left(\frac{1}{k} \operatorname{dist}\left(w_{k} \omega_{j k}(a(0), b(0)), 1\right)\right) .
$$

(This together with (1.9) will imply (1.6).) To do this, consider the mappings

$$
\psi_{j k}(\widetilde{u})=w_{k} \omega_{j k}\left(a\left(k^{-1} \widetilde{u}\right), b\left(k^{-1} \widetilde{u}\right)\right) .
$$

Let $\delta>0, \phi$ be respectively the diffeomorphism and the constant from the Main Lemma (applied to $g=1$, we assume that $\delta<\delta^{\prime}$ ). It follows from the Lemma and (1.11) that $\psi_{j k} \rightarrow \phi$ uniformly with derivatives on $I_{\delta}$, as $k \rightarrow \infty$. Thus, if $k$ is large enough, then the image of $I_{\delta}$ under each $\psi_{j k}$ contains both 1 and $w_{k} \omega_{j k}(a(0), b(0))$, whenever $k$ is large enough. Thus, the distance to 0 of the preimage $\widetilde{u}_{j k}=\psi_{j k}^{-1}(1)$ is $O\left(\operatorname{dist}\left(w_{k} \omega_{j k}(a(0), b(0)), 1\right)\right)$, as $k \rightarrow \infty$. By (1.9), the value $u_{j k}=k^{-1} \widetilde{u}_{j k}$ satisfies (1.12). This together with the previous discussion proves (1.6) and hence, Theorem 1.13. 


\subsection{Approximations of elements and pairs. Approach to Question 2}

For the proof of the statement of Question 2 it would be sufficient to show that for any irrational pair $(A, B)$ there exist a $C=C(A, B)>0$, a sequence of pairs $\left(A_{r}, B_{r}\right)$ and a sequence of words $\omega_{r}(a, b)$ (denote $\left.l_{r}=\left|\omega_{r}\right|\right)$ such that

$$
\omega_{r}\left(A_{r}, B_{r}\right)=1, d_{r}=\operatorname{dist}\left(\left(A_{r}, B_{r}\right),(A, B)\right)<e^{-C l_{r}} .
$$

Let $(a(u), b(u))$ be a conj- nondegenerate deformation of $(A, B),(a(0), b(0))=(A, B)$. As in the previous Subsection, the Main Lemma gives a sequence $w_{k}$ such that the equation $w_{k}(a(u), b(u))=1$ has a solution $u_{k}$ satisfying (1.4), but in general, the distance $\operatorname{dist}\left(w_{k}(A, B), 1\right)$ in (1.4) is no smaller than $e^{-c\left|w_{k}\right|}$. One can fix a $k$ (large enough) and try to multiply $w_{k}$ (from the right) by appropriate auxiliary words $\Omega_{r}, r=1,2, \ldots$ (denote $\left.l_{r}=\left|\Omega_{r}\right|, \omega_{r}=w_{k} \Omega_{r}\right)$, so that

$$
\operatorname{dist}\left(\omega_{r}(A, B), 1\right)<e^{-C^{\prime} l_{r}} \text {, whenever } r \text { is large enough. }
$$

Suppose we deduce then for the new words $\omega_{r}$ that there exists a sequence $u_{r} \rightarrow 0$ such that $\omega_{r}\left(a\left(u_{r}\right), b\left(u_{r}\right)\right)=1$ and (1.4) holds (with $k$ replaced by $r, w_{k}$ by $\omega_{r}, \alpha(0)=(A, B)$ ). Then this together with the previous inequality would imply that $\left(A_{r}, B_{r}\right)=\left(a\left(u_{r}\right), b\left(u_{r}\right)\right)$ satisfy (1.13). The latter deduction could be done, as in the previous Subsection, if the derivatives of the mappings $u \mapsto \Omega_{r}(a(u), b(u)$ ) were uniformly bounded (in $r$ and $k$ ) in one and the same neighborhood of 0 (independent on $r$ and $k$ ). This motivates the following more precise version of Question 5.

Question 8. Let $G$ be a semisimple Lie group, $G_{0}$ be its unity component, $(A, B) \in G \times G$ be an irrational pair. Is it true that for any $R>0$ there exist $\delta^{\prime}=\delta^{\prime}(A, B, R), \Delta=$ $\Delta(A, B, R)>0$ such that for any $g \in G_{0}$, $\operatorname{dist}(g, 1)<R$, there exist $C=C(A, B, g)>0$ and a sequence $\omega_{r}(a, b)$ of words, $\omega_{r}(A, B) \rightarrow g$, such that (denote $\left.l_{r}=\left|\omega_{r}\right|\right)$

1) $\operatorname{dist}\left(\omega_{r}(A, B), g\right)<e^{-C l_{r}}$ for any $r$;

2) the mappings $(a, b) \mapsto \omega_{r}(a, b), G \times G \rightarrow G$, have derivatives of norm less than $\Delta$ in the $\delta^{\prime}$ - neighborhood of the pair $(A, B)$ ?

By the previous discussion, the statement of Question 8 together with the Main Lemma imply the statement of Question 2.

\subsection{Historical remarks}

The famous Tits' alternative $[\mathrm{T}]$ says that any subgroup of linear group satisfies one of the two following incompatible statements:

- either it is solvable up-to-finite, i.e., contains a solvable subgroup of a finite index;

- or it contains a free subgroup with two generators.

Any dense subgroup of a semisimple real Lie group satisfies the second statement: it contains a free subgroup with two generators.

The question of possibility to choose the latter free subgroup to be dense was stated in $[\mathrm{CG}]$ and studied in $[\mathrm{BZ}]$ and $[\mathrm{CG}]$. É.Ghys and Y.Carrière [CG] have proved the positive answer in a particular case. E.Breuillard and T.Gelander [BZ] have proved the positive answer in the general case. 


\section{Proof of the Main Lemma for weakly split Lie groups}

\subsection{Motivation and proof modulo technical details}

A priori, the set of elements $g \in G$ for which the statements of the Main Lemma 1.15] hold is either the whole group $G$, or the empty set. Indeed, it is open (statement 2) of Lemma 1.15). By definition, it is invariant under the left multiplication by words $w(\alpha(0))$. By density of the latters and openness, it is left-shift-invariant, and hence, is either empty, or the whole $G$.

For the proof of the Main Lemma it suffices to fix some appropriately prescribed $g \in G$ and prove its statements for the given $g$. This together with the previous statement will imply the Main Lemma.

Firstly put $g=1$. By density, we can always construct a sequence of words $w_{k}$ so that $W_{k}(0)=w_{k}(\alpha(0)) \rightarrow 1$. In the case, when $a_{i}(0)$ are close to unity, it suffices to take $w_{k}$ to be a sequence of appropriate successive commutators $\omega_{k}$. For the proof of the Main Lemma one has to show that one can achieve that the derivatives of $W_{k}(u)$ at 0 be not too small (more precisely, grow linearly in $k$ ). On the other hand, the previous commutators $\omega_{k}$ converge exponentially to unity and have exponentially decreasing derivatives.

The starting point of the construction of $w_{k}$ with large derivatives is the following ob-

servation. Take some $0<\Delta<\Delta^{\prime}$ and for any $k$ large enough choose a power $\omega_{k}^{m_{k}}$ so that $\Delta<\operatorname{dist}\left(\omega_{k}^{m_{k}}(\alpha(0)), 1\right)<\Delta^{\prime}$. In particular, one can achieve that the sequence $\omega_{k}(\alpha(0))$ converge (passing to a subsequence if necessary; denote $g$ its limit). Then the derivative of the function $\omega_{k}^{m_{k}}(u)$ at 0 in appropriate direction will be large: it will grow linearly in $k$, thus, it will tend to infinity. The simplest one-dimensional analogue of this statement is proved below (in the proof of Proposition 2.3).

For the proof of the Main Lemma we construct

- appropriate words $h(\alpha), g_{i}(\alpha), i=1, \ldots, n$, and put

$$
w_{i 0}=h, w_{i k}=g_{i} w_{i(k-1)} g_{i}^{-1} w_{i(k-1)}^{-1},
$$

- a sequence of collections

$$
\begin{gathered}
M_{k}=\left(m_{1 k}, \ldots, m_{n k}\right), m_{i k} \in \mathbb{N}^{n}, \text { so that the words } \\
w_{k}=w_{M_{k}}=w_{1 k}^{m_{1 k}} \ldots w_{n k}^{m_{n k}} \text { and } g=\lim _{k \rightarrow \infty} w_{k}(\alpha(0))
\end{gathered}
$$

satisfy the statements of the Main Lemma.

In the next Example we do a similar construction in a simple family of (abelian) additive subgroups in $\mathbb{R}$. The proof of the Main Lemma given below uses analogous arguments.

2.1 Example Consider the group $\mathbb{A}_{0}(\mathbb{R})$ of affine automorphisms of $\mathbb{R}$ preserving orientation. This group is generated by multiplications by positive constants and by translations. The subgroup of translations in $\mathbb{A}_{0}(\mathbb{R})$ is canonically identified with $\mathbb{R}$ and will be denoted by the same symbol $\mathbb{R}$. Define the following family of subgroups $\Gamma(s) \subset \mathbb{R} \subset \mathbb{A}_{0}(\mathbb{R})$ :

$$
g(s): x \mapsto s x, s>0, t_{1}: x \mapsto x+1, g(s), t_{1} \in \mathbb{A}_{0}(\mathbb{R}), \quad \Gamma(s)=<g(s), t_{1}>\cap \mathbb{R} .
$$

More generally, for any $u \in \mathbb{R}$ denote

$$
t_{u}: x \mapsto x+u, \quad\left(t_{u} \in \mathbb{R} \subset \mathbb{A}_{0}(\mathbb{R})\right) .
$$


2.2 Remark For any $s>0$ the group $\Gamma(s)$ contains the elements $t_{m s^{k}}, m \in \mathbb{Z}, k \in \mathbb{N} \cup 0$, since it contains $t_{s^{k}}=g(s)^{k} \circ t_{1} \circ g(s)^{-k}$. In fact, $\Gamma(s)=<t_{m s^{k}} \mid m, k \in \mathbb{N} \cup 0>$. The following statement is well-known.

2.3 Proposition Let $\Gamma(s) \subset \mathbb{R}$ be the subgroup family from (2.3). For any $s_{0} \in(0,1)$ there is a sequence $s_{k} \rightarrow s_{0}$ such that for each $k>0$ the group $\Gamma(s), s=s_{k}$, has an extra relation $\tau_{m_{k} s^{k}}=\tau_{1}, m_{k} \in \mathbb{N}$. The same statement holds for nonzero complex parameter values $s_{0}$, $\left|s_{0}\right|<1$, and the corresponding family $\Gamma(s) \subset \mathbb{C}$ of subgroups in the complex affine group.

Proof Let us prove the statement of the Proposition in the real case (the proof in the complex case is analogous with minor changes). For any $k$ take $m_{k}=\left[s_{0}^{-k}\right]$, thus, $m_{k}$ is the integer number such that $m_{k} s_{0}^{k}$ gives a best approximation of 1 with rate less than $s_{0}^{k} ; m_{k} s_{0}^{k} \rightarrow 1$. The values $s_{k}$ we are looking for are the positive solutions to the equation $m_{k} s^{k}=1$ (they correspond to the previous relations by definition). Indeed, it suffices to show that $s_{k} \rightarrow s_{0}$, or equivalently, that the solutions $u_{k}$ of the equations $\psi_{k}(u)=m_{k}\left(s_{0}+u\right)^{k}=1$ converge to 0 . The mapping $\psi_{k}$ is a composition of the homothety $u \mapsto \widetilde{u}=k u$ and the mapping $\phi_{k}: \widetilde{u} \mapsto m_{k}\left(s_{0}+k^{-1} \widetilde{u}\right)^{k}$. One has

$$
\phi_{k}(\widetilde{u})=m_{k} s_{0}^{k}\left(1+k^{-1} \frac{\widetilde{u}}{s_{0}}\right)^{k} \rightarrow \phi(\widetilde{u})=e^{\frac{\widetilde{u}}{s_{0}}}, \text { as } k \rightarrow \infty
$$

The latter limit $\phi(\widetilde{u})$ is a local diffeomorphism near 0 with unit value at 0 , hence, the solutions $\widetilde{u}_{k}$ of the equations $\phi_{k}(\widetilde{u})=1$ converge to 0 . Therefore, so do $u_{k}=k^{-1} \widetilde{u}_{k}$. (moreover, $\left.\left|s_{k}-s_{0}\right|=\left|u_{k}\right|=O\left(\frac{1}{k}\left|s_{0}\right|^{k}\right)\right)$. This proves the Proposition.

As it is shown below, the Main Lemma is implied by the following

2.4 Lemma Let $G$ be a weakly split Lie group, $n=\operatorname{dim} G$. Let $\alpha(u)=\left(a_{1}, \ldots, a_{M}\right)(u), u \in$ $\mathbb{R}^{n}$, be an analytic $\left(C^{\infty}\right)$ conj- nondegenerate family at $u=0$. Let the subgroup $\langle\alpha(0)\rangle \subset G$ be dense. Then there exist

- words $w_{i k}\left(a_{1}, \ldots, a_{M}\right), i=1, \ldots, n, k \in \mathbb{N}$,

- a collection $s_{i}(u), i=1, \ldots, n$, of analytic $\left(C^{\infty}\right)$ functions having the maximal rank $n$ at $u=0,0<s_{i}(0)<1$,

- a basis $v_{1}, \ldots, v_{n}$ of the Lie algebra $T_{1} G$ such that

$$
w_{i k}(\alpha(u))=\exp \left(s_{i}^{k}(u) v_{i k}(u)\right), v_{i k}(u) \rightarrow \widetilde{v}_{i}(u), \text { as } k \rightarrow \infty, \widetilde{v}_{i}(0)=v_{i}
$$

(the latter convergence is $C^{1}$ - uniform on a neighborhood of 0 , the vector functions $\widetilde{v}_{i}(u)$ are analytic (respectively, $C^{\infty}$ ),

$$
l_{i k}=\left|w_{i k}\right|<e^{C k}, C \text { is independent on } k .
$$

The Lemma is proved in the next two Subsections.

Proof of Lemma 1.15 for weakly split Lie groups Let $w_{i k}, v_{i}$ be as in the previous Lemma. The words $w_{k}$ we are looking for will be of type (2.2). Let us construct the corresponding exponents $m_{j k}$.

The vectors $v_{i}$ form a basis, hence, the mapping $\left(t_{1}, \ldots, t_{n}\right) \mapsto \exp \left(t_{1} v_{1}\right), \ldots, \exp \left(t_{n} v_{n}\right)$ is a 1-to-1 mapping of a neighborhood of 0 in the real $t$ - space onto a neighborhood $V \subset G$ of unity. This defines a coordinate system on $V$. (We assume that $V$ is thus parametrized by 
a domain containing the closed cube $\left|t_{i}\right| \leq \varepsilon, \varepsilon>0$; eventually, we choose $\varepsilon$ small enough.) For any $i=1, \ldots, n$ and any $k$ put $m_{i k}=\left[\varepsilon s_{i}^{-k}(0)\right]$. By construction, $m_{i k} s_{i}^{k}(0) \rightarrow \varepsilon$, thus,

$$
w_{M_{k}}(\alpha(0)) \rightarrow g=\prod_{i} \exp \left(\varepsilon v_{i}\right)
$$

Let us prove statement 1) of Lemma 1.15 for the above $g$, if $\varepsilon$ is small enough. Put $\widetilde{u}=k u$. We have to show that the mapping $W_{M_{k}}: \widetilde{u} \mapsto w_{M_{k}}\left(\alpha\left(k^{-1} \widetilde{u}\right)\right)$ converges to a diffeomorphism. Indeed,

$$
\begin{gathered}
W_{M_{k}}(\widetilde{u})=\prod_{i} \exp \left(m_{i k} s_{i}^{k}\left(k^{-1} \widetilde{u}\right) v_{i k}\left(k^{-1} \widetilde{u}\right)\right), v_{i k}\left(k^{-1} \widetilde{u}\right) \rightarrow v_{i}, \text { as } k \rightarrow \infty \text {, by (2.5), } \\
m_{i k} s_{i}^{k}\left(k^{-1} \widetilde{u}\right)= \\
\left.m_{i k} s_{i}^{k}(0)\left(1+k^{-1}\left(d \ln s_{i}(0)\right) \widetilde{u}+o\left(k^{-1}\right)\right)^{k} \rightarrow \varepsilon e^{\left(d \ln s_{i}(0)\right) \widetilde{u}}, \text { as in (2.4) }\right) \text { Thus, } \\
W_{M_{k}}(\widetilde{u}) \rightarrow \phi(\widetilde{u})=\exp \left(\varepsilon e^{\left(d \ln s_{1}(0)\right) \widetilde{u}} v_{1}\right) \ldots \exp \left(\varepsilon e^{\left(d \ln s_{n}(0)\right) \widetilde{u}} v_{n}\right) .
\end{gathered}
$$

Consider the derivative of the mapping $\phi$ at 0 , which is a linear operator $T_{0} \hat{U} \rightarrow T_{\phi(0)} G$. The left shift by $\phi(0)^{-1}$ transforms it to a linear operator $T_{0} \hat{U} \rightarrow T_{1} G$. If $\varepsilon$ is small, then the latter operator is $O\left(\varepsilon^{2}\right)$ - close to $\varepsilon \sum_{i=1}^{n} v_{i}\left(d \ln s_{i}(0)\right): T_{0} \hat{U} \rightarrow T_{1} G$, which is a nondegenerate linear operator: the functions $\ln s_{i}$ have the maximal rank $n$ at 0 , as do $s_{i}$, and the vectors $v_{i}$ are linearly independent. Hence, $\phi$ is a local diffeomorphism. This proves statement 1) of the Main Lemma. By construction, $g=\phi(0)$, which proves the statement 2) of Lemma 1.15. The statement of the Addendum to the Lemma (with $S^{k}>(n+1) \max _{i} s_{i}^{k}(0)$, e.g., $S=\max _{i} s_{i}(0)\left(1+\varepsilon^{\prime}\right)$, with $\varepsilon^{\prime}>0$ chosen arbitrarily small independent on $k$ ) also follows immediately from the construction and (1.4).

Let us prove the statement 3), which says that the lengths $l_{k}$ of the words $w_{k}$ grow at most exponentially, as $k \rightarrow \infty$. By (2.6), this is true for the length $l_{i k}=\left|w_{i k}\right|$. By definition, for any $k$ one has $l_{k} \leq n \max _{i} m_{i k} \max _{i} l_{i k}$,

$$
m_{i k} \leq \frac{\varepsilon}{s_{i}^{k}(0)}<e^{c^{\prime} k}, c^{\prime} \text { is independent on } k .
$$

This together with the previous inequality proves the statement 3). Lemma 1.15 and its Addendum are proved (modulo Lemma 2.4) in the case, when $G$ is weakly split.

\subsection{The iterated commutators $w_{i k}$. The sketch of the proof of Lemma 2.4}

For any element $g \in U \subset G$ (see Definition 1.12) put

$$
s(g)=\text { the eigenvalue of } A d_{g}-I d \text { with the maximal module; denote } v_{g} \text { the eigenvector. }
$$

The value $s(g)$ and the vector $v_{g}$ are well-defined. We consider the iterated commutators by $g$. Namely, for any $g \in G$ define the commutator mapping $\phi_{g}: G \rightarrow G$ :

$$
\phi_{g}(h)=g h g^{-1} h^{-1} \text {. }
$$

In the proof of Lemma 2.4 we use the fact that $s(g)$ is the highest Lyapunov exponent of the commutator mapping $\phi_{g}$ : 
2.5 Proposition Let $G$ be a Lie group, $g \in G$. The derivative at unity of the mapping $\phi_{g}$ is equal to $A d_{g}-I d$. In particular, its eigenvalue with maximal module (if uniquely defined) is equal to $s(g)$; the corresponding eigenvector is $v_{g}$.

2.6 Corollary Let $G$ be a Lie group, $g \in G$ be an element such that $s(g)$ be uniquely defined and $|s(g)|<1$. Then the mapping $\phi_{g}$ is contracting to unity and its germ $(G, 1) \rightarrow(G, 1)$ is conjugated by local analytic coordinate change to a mapping $\left(\mathbb{R}^{n}, 0\right) \rightarrow\left(\mathbb{R}^{n}, 0\right), \mathbb{R}^{n}=\mathbb{R} \times \mathbb{R}^{n-1}$, of the following type (let $x_{1}, x_{2}$ be respectively the coordinates on $\mathbb{R}$ and $\mathbb{R}^{n-1}$ ):

$$
\left(x_{1}, x_{2}\right) \mapsto\left(s(g) x_{1}, Q\left(x_{1}, x_{2}\right)\right), Q\left(x_{1}, 0\right) \equiv 0 .
$$

In particular, it has the unique strong contracting invariant hypersurface $\Sigma_{g}=\left\{x_{1}=0\right\}$ (passing through the unity) transversal to $v_{g}$, see Fig.1. For any $x \in G \backslash \Sigma_{g}$ close to unity

$$
\phi_{g}^{k}(x)=\exp \left(s^{k}(g)\left(c v_{g}+o(1)\right)\right), \text { as } k \rightarrow \infty, c=c(x, g) \neq 0 \text { is independent on } k \text {. }
$$

The derivative of the latter $o(1)=o(1)(x, g)$ in the parameters of $(x, g)$ is uniformly bounded (in $k$ ) on some neighborhood of $(x, g)$ (independent on $k$ ) and tends to zero on the latter neighborhood, as $k \rightarrow \infty$.

The Corollary (without the equality $Q\left(x_{1}, 0\right) \equiv 0$ ) follows from a version of the PoincaréDulac theorem [A]. The local one-parametric subgroup tangent to $v_{g}$ is invariant under $\phi_{g}$. Choosing the latter subgroup to be the $x_{1^{-}}$axis yields $Q\left(x_{1}, 0\right) \equiv 0$.

\section{The dynamics of $\phi_{\mathrm{g}}$}

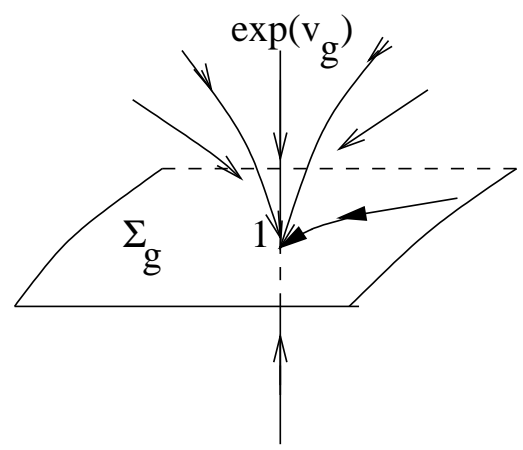

Figure 1:

For the proof of Lemma 2.4 we show (in the next Lemma) that in the conditions of Lemma 2.4 there exist $n$ words $g_{i}\left(a_{1}, \ldots, a_{M}\right), i=1, \ldots, n$, such that the functions

$$
s_{i}(u)=s\left(g_{i}(\alpha(u))\right), i=1, \ldots, n,
$$

are well-defined (locally at 0 ) and have the maximal rank $n$ at 0 . This is the main technical part of the proof of Lemma 2.4. We show that one can achieve that

$$
0<s_{i}(0)<1 \text { and the vectors } v_{i}=v_{g_{i}(\alpha(0))} \text { be linearly independent. }
$$


Afterwards the words $w_{i k}$ are constructed as follows. Consider the strong contracting hypersurfaces of $\phi_{g_{i}(\alpha(0))}$. Let $h$ be a word such that its value $h(\alpha(0))$ lies outside the previous hypersurfaces and is attracted to unity under each mapping $\phi_{g_{i}(\alpha(0))}, i=1, \ldots, n$. Then the corresponding words $w_{i k}$ defined by recurrent formula (2.1) satisfy the statements of Lemma 2.4 Indeed, the asymptotics (2.5) holds with $v_{i}=v_{g_{i}(\alpha(0))}$ by definition and (2.10) (one has to normalize the previous vectors in appropriate way so that the corresponding constants $c$ from (2.10) be equal to 1 ). This proves Lemma 2.4.

2.7 Lemma (Main Technical Lemma). Let $G$ be a Lie group with irreducible adjoint (not necessarily weakly split), $\operatorname{dim} G=n$. Let $\alpha(u)=\left(a_{1}(u), \ldots, a_{M}(u)\right)$ be a family of $M$ ples of its elements depending on the parameter $u=\left(u_{1}, \ldots, u_{n}\right) \in \mathbb{R}^{n}$. Let the subgroup $<\alpha(0)>\subset G$ be dense and $\alpha(u)$ be conj-nondegenerate at 0 . Let $U \subset G$ be an open set and $\sigma: U \rightarrow \mathbb{R}$ be a smooth locally nonconstant function. Then there exist $n$ abstract words $g_{i}\left(a_{1}, \ldots, a_{M}\right), i=1, \ldots, n$, such that the $n$ functions $s_{i}(u)=\sigma\left(g_{i}(\alpha(u))\right)$ are well-defined (locally near 0) and have the maximal rank $n$ at 0 . Moreover, for any given $A_{1}, \ldots, A_{n} \in U$ and $\varepsilon>0$ the previous words $g_{i}$ may be chosen so that $\operatorname{dist}\left(g_{i}(\alpha(0)), A_{i}\right)<\varepsilon$.

Lemma 2.7 will be proved in the next Subsection.

2.8 Remark Let $G$ be a weakly split Lie group, $U$ be the corresponding open subset. For a generic collection of $n$ elements $A_{1}, \ldots, A_{n} \in U$ the corresponding adjoint action eigenvectors $v_{A_{i}}$ (see (2.8) ) are well-defined and linearly independent. One has $0<s\left(A_{i}\right)<1$, whenever $A_{i}$ are close enough to 1 .

The previous Lemma (applied to $\sigma=s(g)$, the above $A_{i}$ and a small $\varepsilon$ ) yields the words

$g_{i}$ we are looking for (satisfying (2.11)). This together with the discussion preceding Lemma 2.7 proves Lemma 2.4

\subsection{Independent eigenvalues. Proof of Lemma 2.7}

Denote $\hat{U}$ the parameter $u$ space under consideration. By assumption, the family $a(u)$ is conj-nondegenerate. This together with the equality of dimensions of $G$ and $\hat{U}$ implies that the derivative along each nonzero vector $v \in T_{0} \hat{U}$ of the function $u \mapsto \operatorname{Conj}(\alpha(u))$ is nonzero. (Fix a $v \in T_{0} \hat{U}$.) The derivatives along $v$ of the mappings $u \mapsto w(\alpha(u))$ form a vector field at the points $w(\alpha(0))$. For the proof of Lemma 2.7 we show (in the next Lemma) that the latter vector field (if well-defined) is not Lipschits at 1 (if it would be Lipschits, it would be given by an infinitesimal automorphism, which would contradict the vanishing of the derivative of $\operatorname{Conj}(\alpha(u)))$. We also show that the lines generated by those derivatives that are large with respect to $\operatorname{dist}(w(\alpha(0)), 1)$ approach any given line in $T_{1} G$, as $w(\alpha(0)) \rightarrow 1$. Then we deduce (in the next Corollary) that one can construct a word with a derivative transversal to level curves of a given function $\sigma$.

2.9 Lemma Let $G$ be a Lie group with irreducible $A d_{G}, \alpha(u)=\left(a_{1}, \ldots, a_{M}\right)(u)$ be a smooth family of $M$-ples of its elements depending on a parameter $u \in \hat{U}=\mathbb{R}^{n}, n=\operatorname{dim} G$. Let the subgroup $\left\langle\alpha(0)>\subset G\right.$ be dense. Let $v \in T_{0} \hat{U}, v \neq 0$, such that the derivative along $v$ of the function $u \mapsto \operatorname{Conj}(\alpha(u))$ does not vanish. Then for any line $\Lambda \subset T_{1} G, 0 \in \Lambda$, there exists a sequence of words $w_{k}\left(a_{1}, \ldots, a_{M}\right), w_{k}(\alpha(0)) \rightarrow 1$, with the following properties: 
1) Consider the derivatives of the functions $w_{k}(\alpha(u))$ along $v$ as a vector field at the points $\left\{w_{k}(\alpha(0))\right\}$. This field is not Lipshits at 1, more precisely,

$$
\frac{\left\|\frac{d w_{k}(\alpha(u))}{d v}\right\|}{\operatorname{dist}\left(w_{k}(\alpha(0)), 1\right)} \rightarrow \infty, \text { as } k \rightarrow \infty .
$$

2) The tangent line to $G$ at $w_{k}(\alpha(0))$ generated by the latter derivative tends to $\Lambda$.

2.10 Corollary In the conditions of the Lemma let $g \in G$ be an arbitrary element, $v \in T_{0} \hat{U}$ be an arbitrary tangent vector. Consider a smooth (locally nonconstant) function $\sigma$ on a neighborhood of $g$ in $G$. Then there exists a sequence of words $\widetilde{w}_{k}, \widetilde{w}_{k}(\alpha(0)) \rightarrow g$, such that the derivatives $\frac{d \widetilde{w}_{k}(\alpha(u))}{d v}$ are transversal to the level hypersurfaces $\sigma=$ const.

The Lemma and its Corollary are proved below.

Proof of Lemma 2.7. Given $\varepsilon>0$ and $A_{1}, \ldots, A_{n} \in U$, let us construct words $g_{i}(\alpha)$, $g_{i}(\alpha(0))$ being $\varepsilon$ - close to $A_{i}$, such that the values $s_{i}(u)=\sigma\left(g_{i}(\alpha(u))\right), i=1, \ldots, n$ are functions of joint rank $n$ at 0 . This will prove Lemma 2.7

Given a tangent vector $v_{1} \neq 0$ to the $u$ - space at 0 , there exists a word $g_{1}$ (denote $\left.s_{1}(u)=\sigma\left(g_{1}(\alpha(u))\right)\right)$ such that $g_{1}(\alpha(0))$ is $\varepsilon$ - close to $A_{1}$ and $\frac{d s_{1}(u)}{d v_{1}} \neq 0(c o n j$ - nondegeneracy and Corollary [2.10). Take another vector $v_{2} \neq 0$ tangent to the level hypersurface of the function $s_{1}$. Again applying the Corollary, one can find a word $g_{2}$ with $g_{2}(\alpha(0)) \varepsilon$ - close to $A_{2}$ such that the derivative along $v_{2}$ of the function $s_{2}: u \mapsto \sigma\left(g_{2}(\alpha(u))\right)$ does not vanish. Now take a vector $v_{3} \neq 0$ tangent to the level surface of the vector function $\left(s_{1}, s_{2}\right)$ and construct a word $g_{3}$ similarly etc. This yields the words $g_{i}$ we are looking for: by construction, the functions $s_{i}: u \mapsto \sigma\left(g_{i}(\alpha(u))\right)$ have rank $n$ at 0 . Lemma 2.7 is proved modulo Lemma 2.9 and Corollary 2.10.

In the proofs of Lemma 2.9 and Corollary 2.10 we use the following notation.

2.11 Definition Let $G$ be a Lie group, $g(u)$ be a family of its elements depending on a parameter $u \in \hat{U}, \hat{U}=\mathbb{R}^{l}$. Let $v \in T_{0} \hat{U}$. Consider the derivative $\frac{d g(u)}{d v}$ of $g(u)$ along $v$, which is a tangent vector to $G$ at the point $g(0)$. The left shift by $g^{-1}(0)$ transforms it to a tangent vector in $T_{1} G$ that is called the translated derivative and denoted $D_{v} g(u)$ :

$$
\frac{d g(u)}{d v}=g(0) D_{v} g(u)
$$

We use the following well-known property of the translated derivative.

2.12 Proposition Let $G$ be a Lie group, $g(u), h(u)$ be a pair of families of its elements depending on a parameter $u \in \hat{U}, v \in T_{0} \hat{U}$. Then

$$
D_{v}(g h(u))=D_{v} h(u)+A d_{h(0)}^{-1} D_{v} g(u) .
$$

Proof of Corollary 2.10. Without loss of generality we consider that $d \sigma(g) \neq 0$ (local nonconstance of $\sigma$ ). By density, it suffices to prove the Corollary for a $g$ represented by some word $w(\alpha(0))$. If the derivative $\frac{d w(\alpha(u))}{d v}$ is already transversal to $\sigma=$ const, then we are done. Suppose now that it is tangent to the level of $\sigma$. Let us modify $w$ to make the derivative transversal. 
Let $\Lambda \subset T_{1} G$ be a line such that its translation image $w(\alpha(0)) \Lambda \subset T_{w(\alpha(0))} G$ is transversal to the level of $\sigma$. Let $w_{k}$ be the corresponding words from the previous Lemma. We claim that the words $\widetilde{w}_{k}=w w_{k}$ are those we are looking for. Indeed, denote

$$
g=w(\alpha(0)), g_{k}=\widetilde{w}_{k}(\alpha(0)) \text {. One has } g_{k} \rightarrow g \text { by construction. }
$$

Let us show that the derivative $\frac{d \widetilde{w}_{k}(\alpha(u))}{d v}$ is transversal to the level of $\sigma$, whenever $k$ is large enough. Indeed, by (2.13),

$$
\begin{gathered}
D_{v} \widetilde{w}_{k}(\alpha(u))=D_{v} w_{k}(\alpha(u))+A d_{w_{k}(\alpha(0))}^{-1} D_{v} w(\alpha(u)) \text {. One has } \\
A d_{w_{k}(\alpha(0))}^{-1}-I d=O\left(\operatorname{dist}\left(w_{k}(\alpha(0)), 1\right)\right)=O\left(\operatorname{dist}\left(g_{k}, g\right)\right)=o\left(D_{v} w_{k}(\alpha(u))\right) \text { by (2.12). } \\
\text { Hence, } D_{v} \widetilde{w}_{k}(\alpha(u))=D_{v} w(\alpha(u))+D_{v} w_{k}(\alpha(u))+o\left(D_{v} w_{k}(\alpha(u))\right) .
\end{gathered}
$$

Consider the differentials $d \sigma(g), d \sigma\left(g_{k}\right)$ as linear forms on $T_{g} G$ and $T_{g_{k}} G$. The left shifts by $g^{-1}$ and $g_{k}^{-1}$ respectively transform them to nonzero linear 1 - forms on $T_{1} G$ whose difference is $O\left(\operatorname{dist}\left(g_{k}, g\right)\right)$. Denote the latter 1- forms by $D \sigma(g)$ and $D \sigma\left(g_{k}\right)$ respectively. For the proof of the Corollary it suffices to show that the value of the form $D \sigma\left(g_{k}\right)$ at the vector $D_{v} \widetilde{w}_{k}(\alpha(u))$ does not vanish, whenever $k$ is large enough. Indeed, let us calculate its value at each term in the right-hand side of (2.14). By definition, $D \sigma(g)\left(D_{v} w(\alpha(u))\right)=0$, hence,

$$
\begin{aligned}
& D \sigma\left(g_{k}\right)\left(D_{v} w(\alpha(u))\right)=O\left(\operatorname{dist}\left(g_{k}, g\right)\right)=o\left(D_{v} w_{k}(\alpha(u))\right), \text { since } D \sigma\left(g_{k}\right)-D \sigma(g)=O\left(\operatorname{dist}\left(g_{k}, g\right)\right) ; \\
& D \sigma\left(g_{k}\right)\left(D_{v} w_{k}(\alpha(u))+o\left(D_{v} w_{k}(\alpha(u))\right)\right)=D \sigma(g)\left(D_{v} w_{k}(\alpha(u))\right)+O\left(\operatorname{dist}\left(g, g_{k}\right)\right) D_{v} w_{k}(\alpha(u)) \\
& +o\left(D_{v} w_{k}(\alpha(u))\right) . \text { Therefore, } D \sigma\left(g_{k}\right)\left(D_{v} \widetilde{w}_{k}(\alpha(u))\right)=D \sigma(g)\left(D_{v} w_{k}(\alpha(u))\right)+o\left(D_{v} w_{k}(\alpha(u))\right) .
\end{aligned}
$$

But by construction and Lemma 2.9. the line generated by the vector $D_{v} w_{k}(\alpha(u))$ tends to the line $\Lambda$, which is transversal to the kernel of the form $D \sigma(g)$. Therefore, there exists a $c>0$ such that for any $k$ large enough the first term in the latter right-hand side has norm greater than $c\left\|D_{v} w_{k}(\alpha(u))\right\|$, and hence, dominates the second term, thus, the right-hand side is nonzero. Corollary 2.10 is proved.

Proof of Lemma 2.9. Firstly we prove statement 1): let us show that one can always find a sequence $w_{k}, w_{k}(\alpha(0)) \rightarrow 1$, so that the corresponding derivatives are not Lipschits at 1 :

$$
\operatorname{dist}\left(w_{k}(\alpha(0)), 1\right)=o\left(\left|\frac{d w_{k}(\alpha(u))}{d v}\right|\right), \text { as } k \rightarrow \infty .
$$

Afterwards, using the irreducibility of the adjoint and density, we deduce that the line generated by the derivative can approach arbitrary given line in $T_{1} G$. This will prove the Lemma.

We prove the first statement by contradiction. Suppose the contrary: the vector field of the previous derivatives of $w(\alpha(u))$ extends to 1 by 0 and is Lipschits at 1, i.e., the derivatives are $O(w(\alpha(0)))$. We claim that it extends up to a vector field on $G$ that is locally Lipschits at each point.

By density, it suffices to prove the Lipschits property along converging sequences of words $w_{k}(\alpha(0))$. More precisely, consider two sequences of words $w_{k}, \widetilde{w}_{k}$ such that $w_{k}(\alpha(0))$ and $\widetilde{w}_{k}(\alpha(0))$ converge to one and the same element $g \in G$. It suffices to show that the previous derivatives of $w_{k}(\alpha(u))$ and $\widetilde{w}_{k}(\alpha(u))$ approach to each other in a Lipschits way. Indeed, consider the ratios $\widetilde{w}_{k}^{-1}(\alpha(u)) w_{k}(\alpha(u))$, whose values at $u=0$ tend to 1 . Therefore, 
by the previous Lipschits assumption at 1 , their derivatives at $u=0$ tend to 0 and are $O\left(\operatorname{dist}\left(\widetilde{w}_{k}^{-1}(\alpha(0)) w_{k}(\alpha(0)), 1\right)\right)=O\left(\operatorname{dist}\left(w_{k}(\alpha(0)), \widetilde{w}_{k}(\alpha(0))\right.\right.$. This implies that the derivatives along $v$ of $w(\alpha(u))$ and $w_{k}(\alpha(u))$ differ by a quantity with a similar asymptotics. The Lipschits property is proved. Thus, the field of derivatives extends up to a locally Lipschits vector field on $G$.

The Lipschits property implies that the flow of the latter field is well-defined (at least locally near 1 ). The vector field agrees with the multiplication, since the same is true at the points $w(\alpha(0)$ ), which are dense. Therefore, its flow is given by (local) automorphisms of $G$. Since $G$ is semisimple, any flow of automorphisms preserves conjugacy classes. Therefore, the function $u \mapsto \operatorname{Conj}(\alpha(u))$ has zero derivative along $v$, - a contradiction.

Now let us prove the second statement of the Lemma. Consider all the sequences $w_{k}$, $w_{k}(\alpha(0)) \rightarrow 1$, that satisfy (2.12). Consider the lines in $T_{w_{k}(\alpha(0))} G$ generated by the derivatives along $v$ of $w_{k}\left(\alpha(u)\right.$ ) and all their limits (along subsequences), which are lines in $T_{1} G$. Denote $\hat{L} \subset T_{1} G$ the union of the limit lines, which is a nonzero set. (If $w_{k}$ is a sequence from (already proved) statement 1), then one can achieve (passing to a subsequence) that the projectivizations of the derivatives converge, and hence, the limit line belongs to $\hat{L}$.) It follows also from definition that $\hat{L}$ is closed.

Below we show that $\hat{L}=T_{1} G$ : this will prove that statement 2) of Lemma [2.9] holds for appropriate sequence $w_{k}$. To do this, we prove firstly that $\hat{L}$ is $A d_{G^{-}}$invariant. Together with the irreducibility of $A d_{G}$, this implies that its linear hull is $T_{1} G$ and for any line $L \in \hat{L}$ there exists a collection of $n-1$ elements $g_{1}, \ldots, g_{n-1} \in G$ such that the lines $L, A d_{g_{1}} L, \ldots, A d_{g_{n-1}} L$ generate $T_{1} G$ as a linear space (we put $g_{0}=1$ ). By density, the $g_{i}$ ' s, $i \geq 1$, can be chosen to be the values $g_{i}=\omega_{i}(\alpha(0))$ of appropriate words $\omega_{i}$. Afterwards, we take arbitrary vector $\nu \in L \backslash 0$. For any collection $r=\left(r_{0}, \ldots, r_{n-1}\right)$ of integer numbers we show that

$$
\text { the line } L_{r}=\mathbb{R}\left(\sum_{i \geq 0} r_{i} A d_{g_{i}} \nu\right) \text { is contained in } \hat{L} \text {. }
$$

This together with the closeness of $\hat{L}$ implies that the whole linear space $T_{1} G$, which is generated by the vectors $A d_{g_{i}} \nu$, coincides with $\hat{L}$.

Let us prove the $A d_{G^{-}}$invariance of $\hat{L}$. By density, it suffices to prove its $A d_{w(\alpha(0))^{-}}$ invariance for any word $w$. Let us fix a $w$. Let $w_{k}, w_{k}(\alpha(0)) \rightarrow 1$, be a sequence satisfying statement (2.12). We assume that the lines generated by the corresponding derivatives converge (denote $L$ the limit line). Consider the new sequence of words $\widetilde{w}_{k}=w w_{k} w^{-1}$. One has $\widetilde{w}_{k}(\alpha(0)) \rightarrow 1$. We claim that the derivatives along $v$ of $\widetilde{w}_{k}(\alpha(u))$ satisfy (2.12) and their lines tend to $A d_{w(\alpha(0))} L$. Indeed, by definition and (2.13),

$$
\begin{gathered}
\frac{d \widetilde{w}_{k}(\alpha(u))}{d v}=\left(\widetilde{w}_{k}(\alpha(0))\right) D_{v} \widetilde{w}_{k}(\alpha(u)), \\
D_{v} \widetilde{w}_{k}(\alpha(u))=A d_{w(\alpha(0))} D_{v} w_{k}(\alpha(u))+O\left(\operatorname{dist}\left(w_{k}(\alpha(0)), 1\right)\right), \text { as } k \rightarrow \infty .
\end{gathered}
$$

The first term in the latter right-hand side is dominant, hence, the line generated by the latter derivative tends to $A d_{w(\alpha(0))} L$. The $A d_{G^{-}}$invariance of $\hat{L}$ is proved.

For the proof of (2.15) consider the following new sequence of words:

$$
\widetilde{w}_{k}=w_{k}^{r_{0}}\left(\omega_{1} w_{k} \omega_{1}^{-1}\right)^{r_{1}} \ldots\left(\omega_{n-1} w_{k} \omega_{n-1}^{-1}\right)^{r_{n-1}} .
$$


We claim that the derivatives along $v$ of the words $\widetilde{w}_{k}$ satisfy (2.12) and the corresponding limit line is $L_{r}$. Indeed,

$$
D_{v} \widetilde{w}_{k}(\alpha(u))=r_{0} D_{v} w_{k}(\alpha(u))+\sum_{i \geq 1} r_{i} A d_{\omega_{i}(\alpha(0))} D_{v} w_{k}(\alpha(u))+O\left(\operatorname{dist}\left(w_{k}(\alpha(0)), 1\right),\right.
$$

as in (2.16). It follows from definition that the sum of the two first terms in the latter righthand side is dominant and its projectivization tends to $L_{r}$. Hence, the same is true for the whole right-hand side. Thus, $\hat{L}=T_{1} G$. Lemma 2.9 is proved.

\section{Case of Lie groups with irreducible adjoint that are not weakly split}

In the case mentioned in the title the proof (given below) of the Main Lemma is essentially the same, as before, but it becomes slightly more technical.

Firstly let us recall the following notation. Given an element $h \in T_{1} G$ of the Lie algebra, its adjoint action $a d_{h}: T_{1} G \rightarrow T_{1} G$ is defined as follows: $v \mapsto[h, v]$. One has

$$
A d_{\exp h}=\exp \left(a d_{h}\right)
$$

In general, the eigenvalues of the adjoint of a generic element $g \in G$ are split into complex conjugated pairs, and the choice of the value $s(g)$ is not unique and should be specified. To do this, we use the following

3.1 Proposition Let $G$ be a semisimple Lie group, $n=\operatorname{dim} G$. Then in appropriate neighborhood of unity in $G$ there exists an open dense subset $V^{\prime}$ (whose complement is an analytic subset in the same neighborhood) such that the adjoint $A d_{g}$ of each $g \in V^{\prime}$ satisfies the following statements:

1) the number of its nonunit complex eigenvalues is maximal and all they are simple;

2) if there is a pair of distinct eigenvalues $\Lambda_{1}, \Lambda_{2} \neq 1$ with $\left|\Lambda_{1}-1\right|=\left|\Lambda_{2}-1\right|$, then $\Lambda_{1}=\bar{\Lambda}_{2}$.

Proof Below we prove similar statements for a generic element $h \in T_{1} G$ of the Lie algebra:

3) the number of nonzero eigenvalues of $a d_{h}$ is maximal and all they are simple;

4) if there is a pair of distinct eigenvalues $\lambda_{1}, \lambda_{2}$ with equal modules, then either $\lambda_{1}=-\lambda_{2}$, or $\lambda_{1}= \pm \bar{\lambda}_{2}$.

Denote $V^{\prime \prime}$ the set of elements $g \in G$ for which statement 1 ) holds. Statement 3) implies already that $V^{\prime \prime}$ is open and dense in a neighborhood of unity (we consider the latter neighborhood as univalently covered by the exponential mapping) and the complement to $V^{\prime \prime}$ is an analytic subset. Consider the set of those $g \in V^{\prime \prime}$ in the latter neighborhood for which there is a pair of eigenvalues $\Lambda_{1}, \Lambda_{2} \neq 1,\left|\Lambda_{1}-1\right|=\left|\Lambda_{2}-1\right|$. If the latter set contains no interior, then statement 2) (and hence, the Proposition) follow immediately. Suppose now it has a nonempty interior, and consider a simply connected subdomain $\widetilde{V}$ of the interior (then each eigenvalue of adjoint is an analytic function on $\widetilde{V})$. We assume that $\left|\Lambda_{1}-1\right| \equiv\left|\Lambda_{2}-1\right|$ on $\widetilde{V}$ (taking a smaller subdomain if necessary). Let us show that $\Lambda_{1} \equiv \bar{\Lambda}_{2}$ on $\widetilde{V}$ (this will imply statement 2)). By statement 4), for any $g \in \widetilde{V}$ (denote $h=\ln g \in T_{1} G, \exp h=g, \lambda_{i}=\ln \Lambda_{i}$ ) either $\lambda_{1}=\bar{\lambda}_{2}$ (hence, the same holds with capitals and we are done), or $\lambda_{1}=-\lambda_{2}$, or 
$\lambda_{1}=-\bar{\lambda}_{2}$. If $\lambda_{1}$ is purely imaginary, and $\lambda_{1}=-\lambda_{2}$, then $\lambda_{1}=\bar{\lambda}_{2}$ (and we are done as before). Let us show that equality $\lambda_{1}=-\lambda_{2}$ cannot hold, if $\lambda_{1}$ is not purely imaginary. Indeed, if it holds, then $\Lambda_{2}=\Lambda_{1}^{-1}$, thus,

$$
\left|\Lambda_{1}-1\right|=\left|\Lambda_{2}-1\right|=\left|\Lambda_{1}^{-1}-1\right|, \text { hence, }\left|\Lambda_{1}\right|=1 \text {. }
$$

This implies that $\lambda_{1} \in i \mathbb{R}$. The impossibility of the other equality $\lambda_{1}=-\bar{\lambda}_{2}$ is proved analogously (then $\lambda_{1} \notin i \mathbb{R}$, since otherwise, one would have $\lambda_{1}=\lambda_{2}$, - a contradiction to the simplicity of eigenvalues). This proves the Proposition modulo statements 3 ) and 4). Let us prove them.

A generic element $h \in T_{1} G$ of the Lie algebra is regular, i.e., has $a d_{h}$ with the nilpotent Jordan block of the minimal possible dimension [VO]. Let $H \subset T_{1} G$ be the corresponding maximal commutative subalgebra containing $h$. The eigenvalues of $a d_{h^{\prime}}, h^{\prime} \in H$, are complexvalued $\mathbb{R}$ - linear functions on $H$, the nonzero ones called roots [VO]. The root decomposition theorem [VO] says that the roots are distinct and someones form an integer basis: each root is an integer linear combination of the basic roots and the latters form a complex basis in the space of complex linear functionals on the complexification of $H$. Thus, the values of nonzero roots are generically distinct, which implies simplicity of the nonzero eigenvalues of $a d_{h^{\prime}}$ for a generic $h^{\prime} \in H$, and hence, statement 3).

Let us prove statement 4$)$. Let $\lambda_{1}(h), \lambda_{2}(h)$ be eigenvalues of $a d_{h}, \lambda_{1} \neq \lambda_{2}$, such that $\left|\lambda_{1}\right| \equiv\left|\lambda_{2}\right|$ on some open set of elements $h$. By linearity, this identity holds on the subalgebra $H$ corresponding to some regular element $h$. Therefore, the restrictions $\left.\lambda_{i}\right|_{H}$ have a common kernel (denote it $K_{\lambda}$ ), which is either a hyperplane, or a plane of codimension two. In the first case it follows that $\lambda_{1}= \pm \lambda_{2}$ and we are done. In the second case they define 1-to- $1 \mathbb{R}$ linear complex functions on the two-dimensional quotient $Q=H / K_{\lambda}$, whose compositional ratio $\left(\lambda_{1}\right)^{-1} \circ \lambda_{2}$ is a real linear function $\mathbb{C} \rightarrow \mathbb{C}$ preserving the module. Therefore, the latter linear function is either multiplication by constant (i.e., $\lambda_{1} \equiv e^{i \theta} \lambda_{2}, \theta=$ const), or a complex conjugation times a complex constant (i.e., $\lambda_{1}=e^{i \theta} \bar{\lambda}_{2}$ ). The constant multiplier $e^{i \theta}$ is equal to \pm 1 in both cases. This follows from the previously mentioned statement on integer basis of roots and the fact that the complex extension of $\bar{\lambda}_{1}$ is also a root. Statement 4 ) and the Proposition are proved.

As before, we define

$$
s(g)=\lambda(g)-1 \text {, where } \lambda(g) \text { is the eigenvalue of } A d_{g} \text { with maximal value of }|\lambda-1| \text {. }
$$

By assumption, the group $G$ is not weakly split. This together with Proposition 3.1 implies that the value $s(g)$ is either uniquely defined and real, or is defined up to complex conjugation and is not real (simplicity of the eigenvalue). The former statement cannot hold for a $g$ that can be chosen arbitrarily close to 1 , since otherwise the group $G$ would be weakly split. Thus, for any $g \in V^{\prime}$ close enough to 1 the value $s(g)$ is not real. We suppose that this is true for all $g \in V^{\prime}$ (choosing the neighborhood of 1 defining $V^{\prime}$ small enough).

Therefore, for any $g \in V^{\prime}$ there is an $A d_{g^{-}}$invariant two-plane in $T_{1} G$ corresponding to the nonreal eigenvalue $\lambda(g)$. Denote the latter plane $L(g)$. It carries an $A d_{g^{-}}$invariant linear complex structure so that $A d_{g}$ acts on $L(g)$ by multiplication by $\lambda(g)$. Hence, the plane $L(g)$ is also $\phi_{g^{-}}$invariant and the restriction to $L(g)$ of the linear part of $\phi_{g}$ is the multiplication by $s(g)$. 
For the proof of the Main Lemma we construct appropriate words $h, g_{i}$ (put $w_{i k}$ to be the corresponding iterated commutators (2.1)), a subsequence $k_{r}$ of the indices $k$, a $n$ - ple of integer numbers $l=\left(l_{1}, \ldots, l_{n}\right)$ and $n$ sequences of natural exponents $M_{r}=\left(m_{1 r}, \ldots, m_{n r}\right)$, put

$$
w_{k_{r}}=w_{M_{r}, k_{r}, l}(\alpha(u))=\left(w_{1, k_{r}+l_{1}}^{m_{1 r}} \ldots w_{n, k_{r}+l_{n}}^{m_{n r}}\right)(\alpha(u)),
$$

so that the corresponding functions

$$
W_{k_{r}}: \widetilde{u} \mapsto w_{k_{r}}\left(\alpha\left(k_{r}^{-1} \widetilde{u}\right)\right)
$$

converge to a local diffeomorphism $\phi: \hat{U} \rightarrow G$. Then the statements of the Main Lemma with $g=\phi(0)$ follow immediately (except for the last statement concerning the lengths of words $w_{k_{r}}$ ). The construction given below yields $\left|m_{i r}\right|=e^{O\left(k_{r}\right)}$, as $r \rightarrow \infty$ (see (3.8). This together with the definition (2.1) of the words $w_{i, k_{r}+l_{i}}$ implies the similar asymptotic bound for $\left|w_{k_{r}}\right|$ and proves the last statement of the Main Lemma.

In the proof of the previous convergence statement we use the following dynamical properties of the mapping $\phi_{g}$.

3.2 Proposition Let $G$ be a Lie group, $\operatorname{dim} G=n, g \in G$ be an element such that the above value $s(g)$ and the plane $L(g)$ are well-defined and $|s(g)|<1, s(g)$ is not real. Then the germ $\phi_{g}:(G, 1) \rightarrow(G, 1)$ is locally conjugated (by analytic diffeomorphism in a neighborhood of unity) to a germ of mapping $\psi:\left(\mathbb{R}^{n}, 0\right) \rightarrow\left(\mathbb{R}^{n}, 0\right), \mathbb{R}^{n}=\mathbb{C} \oplus \mathbb{R}^{n-2}$, of the following type (let $z$ be the coordinate on $\mathbb{C}, y=\left(y_{1}, \ldots, y_{n-2}\right)$ be the coordinates on $\left.\mathbb{R}^{n-2}\right)$ :

$$
\psi(z, y)=(s(g) z, Q(y, z)) .
$$

If the element $g$ depends analytically on a parameter, then so does the conjugating diffeomorphism.

Proposition 3.2 follows from a version of the Poincaré-Dulac theorem [A].

3.3 Corollary In the conditions of the previous Proposition there exists the unique $\phi_{g^{-}}$invariant $n-2$ - surface $\Sigma_{g}=\{z=0\} \subset G$ (strongly contracting invariant surface) passing through 1 transversal to $L(g)$ such that for any $h \in G \backslash \Sigma_{g}$ one has

$$
\begin{gathered}
\phi_{g}^{k}(h)=\exp \left(s^{k}(g) v(g, h)+|s|^{k}(g) \zeta_{k}(g, h)\right), v(g, h) \in L(g), \\
\zeta_{k}(g, h) \rightarrow 0 \text { with derivatives, as } k \rightarrow \infty
\end{gathered}
$$

The vector-functions $v(g, h), \zeta_{k}(g, h)$ are analytic.

Choice of the words $g_{i}$ and the numbers $l_{i}$. We choose words $g_{i}$ so that in particular, $g_{i}(\alpha(0)) \in V^{\prime}$, the functions $s_{i}(u)=s\left(g_{i}(\alpha(u))\right)$ have the maximal real rank $n$ at 0 , and $0<\left|s_{i}(0)\right|<1$. The possibility of choice follows from Lemma 2.7 (applied to $\sigma=s(g)$; it holds for complex-valued functions $\sigma$ as well, which follows immediately from its real version).

For any element $g \in V^{\prime}$ the Lie algebra $T_{1} G$ splits into the direct sum of the plane $L(g)$ and the complementary $A d_{g^{-}}$invariant subspace. Denote $\pi_{g}(h)$ the projection of $T_{1} G$ to $L(g)$ along the complementary invariant subspace.

The statement saying that appropriate sequence of functions (3.2) converges to some function $\phi(\widetilde{u})$ is proved in the same way, as in the previous case, when $G$ is weakly split. To 
show that one can achieve that $\phi$ is a local diffeomorphism, we choose $g_{i}$ and $l=\left(l_{1}, \ldots, l_{n}\right) \in$ $\mathbb{Z}^{n}$ so that $0<\left|s_{i}(0)\right|<1$ and instead of just the maximality of rank of the $s_{i}$ 's, the following more strong statement holds: there exists an $h \in T_{1} G$ such that the linear operator defined by the vector-valued 1 - form

$$
\omega_{h}=\sum_{i=1}^{n}\left(\pi_{g_{i}(\alpha(0))} h\right) s_{i}^{l_{i}-1}(0) d s_{i}(0): T_{0} \hat{U} \rightarrow T_{1} G \text { is an isomorphism. }
$$

The possibility of choice of $g_{i}$ and $l$ will be proved at the end of the Subsection.

3.4 Remark Each term of the previous form is well-defined: $\pi_{g_{i}(\alpha(0))} h \in L\left(g_{i}(\alpha(0))\right)$, and the latter plane $L\left(g_{i}(\alpha(0))\right)$ admits the canonical complex structure defined above; the value of the $i$ - th term of the form on a vector $\psi \in T_{0} \hat{U}$ is defined to be the vector $\pi_{g_{i}(\alpha(0))} h$ times the complex number $s_{i}^{l_{i}-1}(0)\left(d s_{i}(0)\right)(\psi)$. Statement (3.4) implies that the rank of the functions $s_{i}$ at 0 is at least $n$ (and thus, equal to $n$ ).

Choice of the word $h(\alpha)$. For any $h \in T_{1} G$ close to zero, $H=\exp (h) \in G$, one has

$$
\phi_{g_{i}(\alpha(u))}^{k}(H)=\exp \left(s_{i}^{k}(u) v_{i, k, H}(u)\right), v_{i, k, H}(u)=\pi_{g_{i}(\alpha(0))} h+o(h), \text { as } u, h \rightarrow 0, k \rightarrow \infty .
$$

There exists a neighborhood of zero in the parameter space where the derivatives of the vectorfunctions $v_{i, k, H}$ are uniformly bounded. Both latter statements follow from Proposition 3.2 and Corollary 3.3 .

We choose the word $h(\alpha)$ so that statement (3.4) holds with $\pi_{g_{i}(\alpha(0))} h$ replaced by

$$
\nu_{i}=v_{i, k, h(\alpha(0))}: \omega=\sum_{i=1}^{n} \nu_{i} s_{i}^{l_{i}-1}(0) d s_{i}(0): T_{0} \mathbb{R}^{n} \rightarrow T_{1} G \text { is an isomorphism. }
$$

Let us prove the possibility of choice of the word $h(\alpha)$. Statement (3.4) is invariant under multiplication of $h$ by real numbers. Fix an $h \in T_{1} G$ satisfying (3.4). For any $\varepsilon>$ 0 denote $H_{\varepsilon}=\exp (\varepsilon h)$. By density, we can approximate $H_{\varepsilon}$ by a word $h(\alpha(0))$ so that $\operatorname{dist}\left(h(\alpha(0)), H_{\varepsilon}\right)<\varepsilon^{2}$. Then statement (3.6) holds whenever $\varepsilon$ is small enough and $k$ is large enough.

Choice of subsequence $k_{r}$. Let us choose a sequence $k_{r} \rightarrow \infty$ so that

$$
\arg \left(s_{i}^{k_{r}}(0)\right)=k_{r} \arg s_{i}(0) \rightarrow 0(\bmod 2 \pi), \text { as } r \rightarrow \infty, \text { for any } i=1, \ldots, n .
$$

The possibility to do this is proved by considering the real $n$ - torus $\mathbb{T}^{n}=\mathbb{R}^{n} / 2 \pi \mathbb{Z}^{n}$ with the dynamical system of adding the $\arg s_{i}(0)$ to the $i$ - th coordinate and follows from the Poincaré return theorem (see, e.g., [A2]).

Choice of the exponents $M_{r}$. Fix a $\varepsilon>0$ (which will be eventually chosen small enough) and put

$$
m_{i r}=\left[\frac{\varepsilon}{\left|s_{i}\right|^{k_{r}(0)}}\right] \text {. Then by (3.7), } m_{i r} s_{i}^{k_{r}}(0) \rightarrow \varepsilon \text {, as } r \rightarrow \infty \text {. }
$$

Proof of the Main Lemma modulo (3.4). Let $W_{k_{r}}$ be the functions (3.2). One has

$$
W_{k_{r}}(\widetilde{u}) \rightarrow \phi(\widetilde{u})=\exp \left(\varepsilon s_{1}^{l_{1}}(0) e^{\left(d \ln s_{1}(0)\right) \widetilde{u}} \nu_{1}\right) \ldots \exp \left(\varepsilon s_{n}^{l_{n}}(0) e^{\left(d \ln s_{n}(0)\right) \widetilde{u}} \nu_{n}\right), \text { as } r \rightarrow \infty,
$$


as at the end of Subsection 2.1. If $\varepsilon$ is chosen small enough, then $\phi$ is a local diffeomorphism. Indeed, its derivative (left-shifted by $\phi(0)^{-1}$ to $T_{1} G$ ) is $O\left(\varepsilon^{2}\right)$ - close to the $T_{1} G$ - valued 1form

$$
\varepsilon \omega=\varepsilon \sum_{i=1}^{n} s_{i}^{l_{i}-1}(0) \nu_{i} d s_{i}(0): T_{0} \mathbb{R}^{n} \rightarrow T_{1} G .
$$

The latter sum represents a linear isomorphism by (3.6). Thus, the derivative of $\phi$ at 0 is nondegenerate, whenever $\varepsilon$ is small enough. This proves the Main Lemma.

Existence of words $g_{i}$ satisfying (3.4). Recall that in our assumptions the values $s(g)$ are generically not real. Therefore, the corresponding eigenvalues $s(g)+1$ of $A d_{g}$ take either all the nonzero complex values, or only all the values in the unit circle. In both cases $\arg s(g) \not \equiv$ const.

Let $g_{i}(\alpha)$ be a given $n$ - ple of words such that the functions $s_{i}(u)=s\left(g_{i}(\alpha(u))\right)$ have the rank $n$ at 0 and $0<\left|s_{i}(0)\right|<1$. We choose them so that in addition the arguments $\arg s_{i}(0)$ be either irrational multiples of $\pi$, or its rational multiples with large enough denominators (as it will be specified later). This is possible by the last statement of Lemma 2.7 (applied to $A_{i}$ with $\left.\arg s\left(A_{i}\right) \notin \pi \mathbb{Q}\right)$. We will use this assumption in the construction of the numbers $l_{i}$.

We show that we can modify the words $g_{i}$ (by replacing some of them by conjugates $h_{i} g_{j} h_{i}^{-1}$, may be with $j \neq i$ ) so that (3.4) would hold for some $h \in T_{1} G$. (The conjugations do not change the functions $s_{i}$.) To do this, we use the following

3.5 Lemma Let $G$ be a Lie group with irreducible $A d_{G}, n=\operatorname{dim} G$. Let $G_{0}$ be its unity component, $A_{1}, \ldots, A_{n} \in G_{0}$. Consider the corresponding $A d_{A_{i}}$ - invariant 2-planes $L\left(A_{i}\right) \subset$ $T_{1} G$ (we suppose that they are well-defined). Let $m \geq n, \omega_{1}, \ldots, \omega_{n}: \mathbb{R}^{m} \rightarrow \mathbb{C}$ be a $n$-ple of complex-valued linear 1 -forms whose joint real rank is equal to $n$. Then one can choose $n$ elements $h_{1}, \ldots, h_{n} \in G$ and a $n$-ple of indices $1 \leq r_{1}, \ldots, r_{n} \leq n$ (some of the latters may coincide) so that there exist a $n$-ple of vectors $v_{i} \in A d_{h_{i}} L\left(A_{r_{i}}\right)$ such that

$$
\text { the linear operator } \sum_{i=1}^{n} v_{i} \omega_{r_{i}}: \mathbb{R}^{m} \rightarrow T_{1} G \text { is an epimorphism. }
$$

Lemma 3.5 is proved below.

Let us apply Lemma 3.5 to

$$
A_{i}=g_{i}(\alpha(0)), \omega_{i}=d s_{i}(0): T_{0} \mathbb{R}^{n} \rightarrow \mathbb{C}:
$$

it is possible, since the joint real rank of the differentials $d s_{i}(0)$ is equal to $n$. Thus, one can choose $h_{i} \in G$ and an index sequence $r_{1}, \ldots, r_{n}$ (denote $A_{i}^{\prime}=h_{i} g_{r_{i}}(\alpha(0)) h_{i}^{-1}$ ) so that there exist vectors $v_{i} \in L\left(A_{i}^{\prime}\right)=A d_{h_{i}} L\left(g_{r_{i}}(\alpha(0))\right)$ such that the 1 - form

$$
\sum_{i=1}^{n} v_{i} d s_{r_{i}}(0): T_{0} \mathbb{R}^{n} \rightarrow T_{1} G
$$

defines a linear isomorphism. We show that there exists an $h \in T_{1} G$ such that (3.11) is an isomorphism for $v_{i}=s_{r_{i}}^{l_{i}-1}(0) \pi_{A_{i}^{\prime}} h$ (with appropriate integer exponents $l_{i}$ ). Then we can replace $h_{i}$ by close words $h_{i}(\alpha(0))$ (density) so that the previous statement persists. Then (3.4) holds for $g_{i}$ replaced by $h_{i} g_{r_{i}} h_{i}^{-1}$. 
Let us fix the previously chosen $h_{i} \in G$ and $r_{i}$. Then (3.11) is an isomorphism, whenever the collection of the $v_{i}$ ' s belongs to a Zariski open subset (denoted Reg) of the abstract direct sum $\oplus_{i=1}^{n} L\left(A_{i}^{\prime}\right)=\mathbb{C}^{n}$. The complementary set, which consists of the collections of $v_{i}^{\prime}$ 's such that the form (3.11) has rank less than $n$, is an algebraic variety whose degree is bounded by a function in $n$ (denote the latter function by $c(n)$ ). Take arbitrary $h \in T_{1} G$ such that $v_{i}^{\prime}=\pi_{A_{i}^{\prime}} h \neq 0$. It suffices to show that there exists a $l=\left(l_{1}, \ldots, l_{n}\right) \in \mathbb{Z}^{n}$ such that the collection of $v_{i}=s_{r_{i}}^{l_{i}-1}(0) v_{i}^{\prime} \in L\left(A_{i}^{\prime}\right)$ belongs to Reg. To do this, in each plane $L\left(A_{i}^{\prime}\right)$ let us consider the subset $L_{i}^{\prime}=\left\{s_{r_{i}}^{l}(0) v_{i}^{\prime}, l \in \mathbb{Z}\right\}$ and show that the product of the subsets $L_{i}^{\prime}$ intersects Reg.

If all the arguments $\arg s_{i}(0)$ are irrational multiples of $\pi$, then the latter product is Zariski dense in $\oplus_{i} L\left(A_{i}^{\prime}\right)=\mathbb{C}^{n}$ and hence, intersects Reg. If some argument $\arg s_{i}(0)$ is a rational multiple of $\pi$ (denote $m_{i}$ the corresponding denominator), then the Zariski closure of each $L_{i}^{\prime}$ is a union of $m_{i}$ real lines and is an algebraic variety of degree $m_{i}$. If all the $m_{i}$ 's are much larger than $c(n)$, then so is the degree of the product of the sets $L_{i}^{\prime}$. Hence, the latter product is contained in no algebraic variety of degree no greater than $c(n)$, and in particular, intersects Reg. Hence, (3.4) holds for appropriate $l$.

Proof of Lemma 3.5. We show that one can choose $v_{i}$ and $h_{i}$ and a sequence $\omega_{r_{1}}, \ldots, \omega_{r_{n}}$ of the forms $\omega_{i}$ (some indices $r_{i}$ may coincide) so that for any $j=1, \ldots, n$ the 1 - form

$$
\Omega_{j}=\sum_{i=1}^{j} v_{i} \omega_{r_{i}}: \mathbb{R}^{m} \rightarrow T_{1} G \text { has kernel of real codimension at least } j .
$$

This will prove the Lemma.

We prove the previous statement by induction in $j$.

Induction base: statement (3.12) is obvious for $j=1, v_{1} \neq 0$ and $\omega_{r_{1}} \not \equiv 0$.

Induction step: $j>1, j \leq n$. Let we have already chosen $h_{i}, v_{i}, \omega_{r_{i}}, i \leq j-1$, so that (3.12) holds for $j$ replaced by $j-1$ (let $\Omega_{j-1}$ be the corresponding sum of forms). Let us construct $h_{j}, v_{j}$ and $\omega_{r_{j}}$ such that (3.12) holds.

Let $K=K e r \Omega_{j-1}$. By the induction hypothesis, $\operatorname{codim} K \geq j-1$. If $\operatorname{codim} K \geq j$, then (3.12) holds true with $v_{j}=0$ and arbitrary $h_{j}$ and $\omega_{r_{j}}$. Thus, everywhere below we consider that $\operatorname{codim} K=j-1$, in particular, $K \neq 0$. Let us choose $h_{j}$ so that $L=A d_{h_{j}} L\left(A_{j}\right)$ be not contained in the image of the form $\Omega_{j-1}$. One can achieve this by the irreducibility of the adjoint and since $\operatorname{dim}\left(\Omega_{j-1}\left(\mathbb{R}^{m}\right)\right)=\operatorname{codim} K=j-1<n$. It suffices to show that we can choose a vector $v_{j} \in L$ so that $\operatorname{codimKer} \Omega_{j}>\operatorname{codim} K$ : this will prove the induction step. Put

$$
\Lambda=\Omega_{j-1}\left(\mathbb{R}^{m}\right) \cap L \text {. Then } \Lambda \text { is either } 0 \text {, or a real line. }
$$

Since $K \neq 0$, one can choose a form $\omega_{r_{j}}$ (let us fix it), $r_{j}=1, \ldots, n$, so that $\left.\omega_{r_{j}}\right|_{K} \not \equiv 0$.

Case $\Lambda=0$. Then for any $v_{j} \in L \backslash 0$ statement (3.12) holds. Indeed, the kernel $\Omega_{j}=0$ is strictly contained in $K$ (thus, has a larger codimension): the images of the forms $\Omega_{j-1}$ and $v_{j} \omega_{r_{j}}=\Omega_{j}-\Omega_{j-1}$ are nonzero and intersect only at $0(\Lambda=0)$, thus, $\Omega_{j}$ can vanish only in $K$ and exactly at those points of $K$, where $\omega_{r_{j}}=0$. Since $\left.\omega_{r_{j}}\right|_{K} \not \equiv 0$, one has $\operatorname{codimKer} \Omega_{j}>\operatorname{codim} K$.

Case, when $\Lambda$ is a line. Consider the preimage $P=\left(\Omega_{j-1}\right)^{-1}(\Lambda)$. By definition, $\operatorname{codim} P=$ $j-2$. One has

$\operatorname{Ker} \Omega_{j} \subset P$, whatever $v_{j} \in L$ we chose : 
the form $\Omega_{j}$ can vanish only at the vectors where the values of the forms $\Omega_{j-1}$ and $v_{j} \omega_{r_{j}}$ lie in one and the same real line. Consider the restriction $\left.\omega_{r_{j}}\right|_{P}$, whose image is either the plane $\mathbb{C}$, or a line. Suppose that its image is a line. Then we can take $v_{j} \in L$ so that the image of $\left.v_{j} \omega_{r_{j}}\right|_{P}$ will be arbitrarily given line in $L$, in particular, not contained in $\Lambda=\Omega_{j-1}(P)$. Therefore, $\Omega_{j}=0$ only at those vectors where $\Omega_{j-1}=0$ and $\omega_{r_{j}}=0$. Thus, $\operatorname{Ker} \Omega_{j}=K \cap \operatorname{Ker}\left(\left.\omega_{r_{j}}\right|_{P}\right)$, and $\operatorname{codimKer} \Omega_{j}<\operatorname{codim} K$, as in the previous case.

Now suppose that $\omega_{r_{j}}(P)=\mathbb{C}$, thus, $\operatorname{Ker}\left(\left.\omega_{r_{j}}\right|_{P}\right)$ has codimension 2 in $P$. For a given $v_{j} \in L \backslash 0$, denote

$$
P^{\prime}=\left\{v^{\prime \prime} \in P \mid v_{j} \omega_{r_{j}}\left(v^{\prime \prime}\right) \in \Lambda\right\} .
$$

This is a hyperplane in $P$, and hence, it has codimension $j-1$ in $\mathbb{R}^{m}$. In particular, $\left.\omega_{r_{j}}\right|_{P^{\prime}} \not \equiv 0$. It follows from definition that $\operatorname{Ker} \Omega_{j} \subset P^{\prime}$. The kernel $\operatorname{Ker} \Omega_{j}$ is (by definition) the space of solutions of the linear equation $\Omega_{j-1}=-v_{j} \omega_{r_{j}}$ in $P^{\prime}$. Since $\left.\omega_{r_{j}}\right|_{P^{\prime}} \not \equiv 0$, one can modify the vector $v_{j}$ (e.g., by multiplication by a real constant) in such a way that the latter equation be nontrivial. Hence, its solution space is smaller than $P^{\prime}$, and hence, has a larger codimension (at least $j$ ). The induction step is over. Statement (3.12) and Lemma 3.5] are proved.

\section{Proof of Theorem 1.1 for arbitrary Lie group}

We have already proved Theorem 1.1 for any Lie group with $\mathbb{R}$ - irreducible adjoint. Let us prove it for arbitrary Lie group. Without loss of generality we assume that the elements $A, B \in G$ under consideration generate a dense subgroup (passing from $G$ to the closure of the subgroup), since any closed subgroup of a Lie group is a Lie subgroup [VO]. Denote $G_{0}$ the unity component of $G$.

Case 1), when $G$ is real-semisimple, connected and simply connected. Then $G=\prod_{i=1}^{k} G_{i}, G_{i}$ are simple. By the above version of Theorem 1.1, we can slightly modify the $i$ - th coordinates $A_{i}, B_{i} \in G_{i}$ of $A, B$ to satisfy some relation in $G_{i}: w_{i}\left(A_{i}, B_{i}\right)=1$. Let us show that the new elements $A$ and $B$ satisfy a relation in $G$. This will prove Theorem 1.1.

Consider the commutator []$_{1}=w_{1} w_{2} w_{1}^{-1} w_{2}^{-1}$. Its value at $(A, B)$ is contained in $\prod_{i=3}^{k} G_{i}$. Indeed, it follows from definition that $w_{i}(A, B) \in \prod_{j \neq i} G_{i}$ and the groups $G_{i}$ commute. Therefore, the $G_{1^{-}}$component of the latter commutator is 1 , and so is the $G_{2^{-}}$component. Analogously, consider the next commutator:

$$
[]_{2}=[]_{1} w_{3}[]_{1}^{-1} w_{3}^{-1}: \text { one has }[]_{2}(A, B) \in \prod_{i \geq 4} G_{i}, \text { etc } \ldots
$$

The commutator []$_{k-1}=[]_{k-2} w_{k}[]_{k-2}^{-1} w_{k}^{-1}$ takes unit value at $(A, B)$. This is a relation we are looking for.

Case 2), when $G$ is semisimple connected but not simply connected. Let $\widetilde{G}$ be its universal covering, which is a product of simple groups, as above. Then the elements of $\widetilde{G}$ projected to $1 \in G$ belong to its center and form a discrete normal subgroup of $\widetilde{G}$. Denote $\widetilde{A}, \widetilde{B} \in \widetilde{G}$ the lifted elements $A$ and $B$. The subgroup $<\widetilde{A}, \widetilde{B}>\subset \widetilde{G}$ is dense. Indeed, it is projected onto a dense subgroup $<A, B>\subset G$. Therefore, its closure (which is a Lie subgroup) should be projected onto $G$, hence, it is $\widetilde{G}$. This reduces us to the previous case: one can perturb slightly $A$ and $B$ in order to have relation between $\widetilde{A}, \widetilde{B} \in \widetilde{G}$ (hence, the same relation will hold for $A$ and $B$ ). 
Case 3), when $G$ is semisimple but not connected. Without loss of generality (as in the previous case) we consider that $G_{0}$ is simply connected, and hence, is a product $\prod_{i=1}^{k} G_{i}$ of connected simple groups $G_{i}$. Consider the adjoint homomorphism $A d: G \rightarrow \operatorname{Aut}\left(T_{1} G\right)$ (by $\operatorname{Aut}\left(T_{1} G\right)$ we denote the group of automorphisms of the Lie algebra $T_{1} G$ ). It defines a (local) isomorphism near 1 of the unity components of $G$ and $\operatorname{Aut}\left(T_{1} G\right)$. (The quotient $H=$ $\operatorname{Aut}\left(T_{1} G\right) / A d\left(G_{0}\right)$ is finite, see [VO].) The decomposition of $T_{1} G$ as a direct sum of simple Lie algebras $T_{1} G_{i}$ is unique up to permutation of isomorphic algebras. This implies that the adjoint of each element of $G$ either preserves the decomposition, or permutes isomorphic algebras $T_{1} G_{i}$. Therefore, the Lie algebra $T_{1} G$ splits as a direct sum

$$
T_{1} G=\oplus_{j=1}^{m} h_{j}
$$

of minimal $A d_{G^{-}}$invariant subspaces $h_{j}$. Each of the latters is either $T_{1} G_{i}$, or a sum of several isomorphic $T_{1} G_{i}$ ' s, thus, each $h_{j}$ is the Lie algebra of the Lie group (denoted $H_{j}$ ) that is a (product of some) $G_{i}$. Hence,

$$
G_{0}=\prod_{j=1}^{l} H_{j}
$$

By construction, each $H_{j}$ is a normal subgroup in $G$, thus, there is a natural homomorphism

$$
\phi: G \rightarrow \prod_{j} \hat{H}_{j}, \hat{H}_{j}=G / \prod_{r \neq j} H_{r}
$$

Each group $\hat{H}_{j}$ is disconnected and its unity component is isomorphic to $H_{j}$ (both the latters are simply connected, since so is $G_{0}$ ). The homomorphism $\phi$ is injective (but not necessarily surjective) and induces an isomorphism of $G_{0}$ and the unity component of the product $\prod_{j} \hat{H}_{j}$. The elements $\phi(A), \phi(B)$ generate a dense subgroup in $\phi(G)$. The group $\phi(G)$ is projected onto each $\hat{H}_{j}$. The adjoint of $G$ acts irreducibly on $h_{j}$, hence, $A d_{\hat{H}_{j}}$ is also irreducible on $T_{1} \hat{H}_{j}$.

Therefore, Theorem 1.1, which is proved in the irreducible case, holds for each $\hat{H}_{j}$. Now we prove Theorem 1.1 in the same way, as for the product of the $\hat{H}_{j}$ ' s, see the previous Case 1): by Theorem 1.1 applied to $\hat{H}_{j}$, modifying slightly the $\hat{H}_{j^{-}}$coordinates of $\phi(A), \phi(B)$ one can achieve that some relations $w_{j}(\phi(A), \phi(B))=1$ hold in $\hat{H}_{j}$, etc.

Case 4): general case. Now let $G$ be a Lie group that is not semisimple. This means that its unity component $G_{0}$ contains a maximal connected solvable normal subgroup (denote it $H$ ) of positive dimension. The subgroup $H$ is unique and is invariant under the automorphisms of $G_{0}$. Hence, it is a normal subgroup in the whole (may be disconnected) group $G$. The quotient $G / H$ is a semisimple Lie group. Therefore, applying the result of Case 3 ) to the quotient yields that modifying slightly $A$ and $B$ one can achieve that the value of some word $w(A, B)$ belongs to $H$. Thus, the intersection $\langle A, B\rangle \cap H$ is nontrivial and is a solvable group. Therefore, the group generated by the perturbed elements $A$ and $B$ cannot be free. The proof of Theorem 1.1 is completed. 


\section{A short proof of Theorem 1.1 for dense subgroups in $G=$ $P S L_{2}(\mathbb{C})$}

Let $A, B \in P S L_{2}(\mathbb{C})$ generate a free dense subgroup. We prove Theorem 1.1 by contradiction. Suppose there is a (simply connected) neighborhood $V \subset P S L_{2}(\mathbb{C}) \times P S L_{2}(\mathbb{C})$ of the pair $(A, B)$ such that each pair $(a, b) \in V$ generates a free subgroup. Thus, each word $w(a, b)$ is a holomorphic function in $(a, b) \in P S L_{2}(\mathbb{C}) \times P S L_{2}(\mathbb{C})$ with values in $P S L_{2}(\mathbb{C})$; distinct words define holomorphic functions with disjoint graphs over $V$. Using holomorphic motion of fixed points of the elements $w(a, b) \in P S L_{2}(\mathbb{C})$, we construct a nonstandard measurable almost complex structure on $\overline{\mathbb{C}}$ invariant under the action of $\langle A, B\rangle$ (and hence, under the action of the whole group $P S L_{2}(\mathbb{C})$ by density). But the only measurable almost complex structure preserved under the action of $P S L_{2}(\mathbb{C})$ on $\overline{\mathbb{C}}$ is the standard complex structure - a contradiction.

5.1 Remark The author's initial proof of Theorem 1.1 in the case, when $G=P S L_{2}(\mathbb{C})$, followed a similar scheme (using holomorphic motion of fixed points) but was longer than the one presented below. The final quasiconformal mapping argument, which simplified the proof essentially, is due to Étienne Ghys.

Recall that an element $b \in P S L_{2}(\mathbb{C})$ is called elliptic, if its action on $\overline{\mathbb{C}}$ is conjugated to a rotation. It is called hyperbolic, if it has two fixed points: one attracting and the other one repelling. Otherwise it is parabolic, i.e., has a unique fixed point and is conjugated to the translation.

The liberty stability assumption implies that the elements $w(a, b) \in P S L_{2}(\mathbb{C})$ are hyperbolic whenever $(a, b) \in V$ : in other terms, the multipliers of their fixed points have modules different from 1. Indeed, their fixed points are holomorphic functions in $(a, b) \in V$ (may be multivalued with possible double branchings corresponding to the parabolic elements $w(a, b)$ ), and so are the multipliers of the fixed points. Suppose the multiplier $\mu(a, b)$ of a fixed point of $w(a, b)$ has a unit module. Then one can find points $\left(a^{\prime}, b^{\prime}\right)$ arbitrarily close to $(a, b)$ where $\mu\left(a^{\prime}, b^{\prime}\right)$ is equal to nontrivial roots of unity (the holomorphic mappings are open). This means that $w\left(a^{\prime}, b^{\prime}\right) \in P S L_{2}(\mathbb{C})$ is a nontrivial element of finite order - a contradiction to the liberty stability assumption.

Thus, each element $w(a, b) \in P S L_{2}(\mathbb{C}),(a, b) \in V$, is hyperbolic, hence, its fixed points are analytic functions in $(a, b) \in V$. The graphs of the fixed point functions are disjoint. Indeed, otherwise, if two distinct hyperbolic elements of $P S L_{2}(\mathbb{C})$ have one common fixed point, then their commutator is parabolic: the latter fixed point is its unique fixed point. This contradicts the hyperbolicity of the commutator.

In other words, the previous fixed point families form a holomorphic motion over $V$ of the fixed points of the elements $w(A, B)$. The latter elements are dense by assumption, hence, so are their fixed points. The previous holomorphic motion extends up to a holomorphic motion of the Riemann sphere: this means that one can extend the collection of graphs of fixed points of $w(a, b)$ up to filling-in the product $V \times \overline{\mathbb{C}}$ by disjoint graphs of holomorphic functions on $V$ with values in $\overline{\mathbb{C}}$. This follows immediately from density and an elementary normality argument (e.g., a version of Montel's theorem, see [L]).

5.2 Remark The well-known Slodkowski theorem $[\mathrm{S}]$ says that any holomorphic motion in $D \times \overline{\mathbb{C}}$ of any subset of the Riemann sphere over unit disc $D$ extends up to a holomorphic 
motion of the whole Riemann sphere. Here we do not use this theorem in full generality.

It is well-known (see, e.g., [ST]) that any holomorphic motion has a quasiconformal holonomy. More precisely, in our case this means the following. For any $(a, b) \in V$ consider the mapping $h_{a, b}: \overline{\mathbb{C}} \rightarrow \overline{\mathbb{C}}$ defined to send the fixed points of $w(a, b)$ to those of $w(A, B)$. The mapping $h_{a, b}$ extends up to a quasiconformal homeomorphism of $\overline{\mathbb{C}}$ (depending holomorphically on the parameters $(a, b))$. The quasiconformal homeomorphism $h_{a, b}$ transforms the standard complex structure on $\overline{\mathbb{C}}$ to a measurable almost complex (denoted by $\sigma(a, b)$ ). It follows from construction that $\sigma(a, b)$ is invariant under the group $\langle A, B\rangle$, and hence, under $P S L_{2}(\mathbb{C})$ by density. Now to prove the Theorem, it suffices to show that for a generic pair $(a, b)$ the almost complex structure $\sigma(a, b)$ is not standard.

For any $(a, b) \in V$ the elements $a$ and $b$ are hyperbolic with distinct fixed points; the latters form a quadruple denoted $Q(a, b)$ of points in $\overline{\mathbb{C}}$. If the cross-ratios of two quadruples $Q(a, b)$ and $Q(A, B)$ are distinct, then the quasiconformal homeomorphism $h_{a, b}$, which sends $Q(a, b)$ to $Q(A, B)$, is not conformal; hence, $\sigma(a, b)$ is not standard. This together with the discussion at the beginning of the Section proves Theorem 1.1.

\section{Proof of Theorem 1.13}

Let us construct the words $\omega_{j k}$ satisfying (1.9)-(1.11). This together with the discussion in 1.4 will prove Theorem 1.13, Denote

$$
x_{k}=w_{k}^{-1}(A, B)
$$

Consider the words $w_{i k}$ from Lemma 2.4 (chosen as specified below, let us fix them) and construct $\omega_{j k}$ as appropriate products of powers of some conjugates of $w_{1 k}$ (some powers and conjugates may repeat, but the list of different powers and different conjugating words in the product will be independent on $k$ ).

Let $s_{i}(u)=s\left(g_{i}(\alpha(u))\right)$ be the functions from Lemma 2.4 corresponding to the chosen words $w_{i k}, v_{i k}(u), \widetilde{v}_{i}(u), v_{i}$ be the corresponding vector functions and vector from (2.5). Denote

$$
S=\max _{i} s_{i}(0) ; \text { one has } 0<S<1 .
$$

Without loss of generality we assume that $S=s_{1}(0)$ : one can achieve this by changing the numeration by $i$ of the words $w_{i k}$.

Below we use the following notations: for a vector $h \in T_{1} G$ of the Lie algebra with $\mathbb{R}$ diagonalizable adjoint by $s(h)$ (respectively, $v_{h}$ ) we denote the maximal eigenvalue of $a d_{h}$ (respectively, the corresponding eigenvector). This agrees with the previous definition of $s(g)$ for $g \in G$ as follows:

$$
s(\exp h)=e^{s(h)}
$$

Firstly let us motivate the construction. Let $g_{i}, h$ be the words from Lemma 2.7 (the words $w_{i k}$ are the corresponding iterated commutators (2.1)). For any fixed $k^{\prime} \in \mathbb{N}$ the products

$$
w_{1 k^{\prime}}^{m_{1 k^{\prime}}}(A, B) \ldots w_{n k^{\prime}}^{m_{n k^{\prime}}}(A, B), m_{i k^{\prime}} \in \mathbb{Z}
$$

with "not too big" powers $m_{j k^{\prime}}$ form a "local $n$ - dimensional lattice" (denoted $\Gamma_{k^{\prime}}$ ) with steps of length no greater than $c S^{k^{\prime}}, c>0$ is a constant independent on $k^{\prime}$. This follows from (2.5). 
We wish to construct approximation of $x_{k}$ with accuracy of order $S^{k^{2}}$, so we can use the words of the lattice $\Gamma_{k^{2}}$, whose step is of a similar order. But its generating words $w_{i k^{2}}$ have lengths greater than $2^{k^{2}}$. Therefore, if an approximating word $\omega_{k k}$ contains some of them, it will be too long and will not satisfy (1.10).

Below we firstly modify the lattices $\Gamma_{j k}$ in order to diminish the lengths of their generating words (making them $e^{O(k)}$ ) keeping their steps $O\left(\widetilde{s}^{j k}\right), 0<\widetilde{s}<1$. Afterwards we can already approximate $x_{k}$ by a word $\omega_{j k}(A, B) \in \Gamma_{j k}$, but its length will be too big, namely, $e^{O(j k)}$. To diminish the length, we approximate $x_{k}$ subsequently by words $\omega_{j k}(A, B)$ similarly to $\widetilde{s}^{-1}$ - ary sums $\sum_{l=1}^{j} r_{l} \widetilde{s}^{l}, 0<r_{l}<\widetilde{s}^{-1}$, approximating a given real number. It is done inductively in $j$ as follows (the details are given below). First approximate $x_{k}$ by a word $\omega_{2 k}(A, B) \in \Gamma_{2 k}$. Put $x_{2 k}=\omega_{2 k}^{-1} x_{k}$. Approximate $x_{2 k}$ by a word $\omega_{3 k}^{\prime}(A, B) \in \Gamma_{3 k}$ and put $\omega_{3 k}=\omega_{2 k} \omega_{3 k}^{\prime}$. Analogously put $x_{3 k}=\omega_{3 k}^{-1} x_{k}$, approximate it by $\omega_{4 k}^{\prime}(A, B) \in \Gamma_{4 k}$ and put $\omega_{4 k}=\omega_{3 k} \omega_{4 k}^{\prime}$, etc.

To modify the lattices $\Gamma_{j k}$, as specified above, we first construct auxiliary words $h_{1}=$ $1, h_{2}, \ldots, h_{n}, R_{1}, \ldots, R_{n}, n^{2}$ integer numbers $r_{i j}, i, j=1, \ldots, n$, and put

$$
\begin{gathered}
p_{i k}=\left(h_{1} w_{1 k} h_{1}^{-1}\right)^{r_{i 1}} \ldots\left(h_{n} w_{1 k} h_{n}^{-1}\right)^{r_{i n}}, i=1, \ldots, n, k \in \mathbb{N}, \\
q_{1 ; i k}=p_{i k} R_{i} p_{i k}^{-1} R_{i}^{-1}, q_{j ; i k}=p_{i k} q_{j-1 ; i k} p_{i k}^{-1} q_{j-1 ; i k}^{-1} .
\end{gathered}
$$

(For each $j, k \in \mathbb{N}$ the words $q_{j ; i k}(A, B), i=1, \ldots, n$, play the role of "generators" of the previously mentioned modified lattice $\Gamma_{j k}$.) We do our construction so that

$$
\begin{gathered}
q_{j, i k}(a(u), b(u))=\exp \left(s_{i k}^{j}(u) v_{j ; i k}(u)\right), s_{i k}(u)=s_{1}^{k}(u) c_{i k}(u), \\
c_{i k} \rightarrow c_{i}(u)>0, v_{j ; i k}(u) \rightarrow V_{i}(u), \text { as } j, k \rightarrow \infty
\end{gathered}
$$

all the latter (vector) functions are $C^{\infty}$ and the last convergences are uniform with derivatives in a neighborhood of 0 (independent on $j$ and $k$ ),

$$
\text { the vectors } V_{i}(0) \text { form a basis in } T_{1} G \text {. Put } \widetilde{V}=2 n \max _{i}\left|V_{i}(0)\right| \text {. }
$$

(The latter norm correspond to a fixed left-invariant metric on $G$.) Choosing $k$ large enough one can always achieve that $2 \widetilde{V} s_{i k}(0)<1$. Without loss of generality we consider that $c_{1}(0) \geq c_{i}(0)$ for any $i \neq 1$, thus, $s_{1 k}(0) \geq s_{i k}(0)$ whenever $i \neq 1$.

In what follows, we construct the words $\omega_{j k}$ we are looking for by induction in $j$ so that $\omega_{j k}(A, B)$ approximate $x_{k}$ with the accuracy less than $\widetilde{V}\left(2 s_{1 k}(0)\right)^{j}$. This together with (6.4) will imply (1.9). The upper bounds (1.10) and (1.11) of lengths and derivatives will be proved afterwards.

Induction base: the construction of $\omega_{2 k}$. Put

$$
\omega_{2 k}=q_{2 ; 1 k}^{r_{2 ; 1 k}} \ldots q_{2 ; n k}^{r_{2 ; n k}},
$$

where $r_{2 ; i k}$ are chosen as follows. Let us write down

$$
x_{k}=\exp \left(\tau_{2 ; 1 k} v_{2 ; 1 k}(0)\right) \ldots \exp \left(\tau_{2 ; n k} v_{2 ; n k}(0)\right) . \text { Put } r_{2 ; i k}=\left[\tau_{2 ; i k} s_{i k}^{-2}(0)\right] .
$$

It follows from definition that $\omega_{2 k}(A, B)$ approximates $x_{k}$ with the accuracy at most $\widetilde{V} s_{1 k}^{2}(0)(1+o(1))<2 \widetilde{V} s_{1 k}^{2}(0)$, whenever $k$ is large enough. 
Induction step: the construction of the words $\omega_{j k}, j>2$. Let the word $\omega_{j k}$ be already constructed. Let us construct the word $\omega_{j+1, k}$. Put

$$
x_{j k}=\omega_{j k}^{-1}(A, B) x_{k} .
$$

We already know (the induction hypothesis) that $\omega_{j k}(A, B)$ approximates $x_{k}$ with accuracy $\widetilde{V}\left(2 s_{1 k}(0)\right)^{j}$, and our goal is to make it at most $\widetilde{V}\left(2 s_{1 k}(0)\right)^{j+1}$. To do this, we construct another word $\omega_{j+1, k}^{\prime}(A, B)$ approximating $x_{i k}$ with the accuracy $\widetilde{V} s_{1 k}(0)\left(2 s_{1 k}(0)\right)^{j}(1+o(1))$ $($ as $k \rightarrow \infty)$ and put

$$
\omega_{j+1, k}=\omega_{j k} \omega_{j+1, k}^{\prime}
$$

Then by construction, the word $\omega_{j+1, k}(A, B)$ approximates $x_{k}$ with asymptotically the same accuracy, which will be less than $\widetilde{V}\left(2 s_{1 k}(0)\right)^{j+1}$, whenever $k$ is large enough. Namely, put

$$
\omega_{j+1, k}^{\prime}=q_{j+1 ; 1 k}^{r_{j+1 ; 1 k}} \ldots q_{j+1 ; n k}^{r_{j+1 ; n k}}
$$

where $r_{j+1 ; i k}$ are chosen as follows. Let us write down

$$
x_{j k}=\exp \left(\tau_{j+1 ; 1 k} v_{j+1 ; 1 k}(0)\right) \ldots \exp \left(\tau_{j+1 ; n k} v_{j+1 ; n k}(0)\right) . \text { Put } r_{j+1 ; i k}=\left[\tau_{j+1 ; i k} s_{i k}^{-(j+1)}(0)\right] .
$$

The desired rate of approximation of $x_{i k}$ by $\omega_{i+1, k}^{\prime}$ follows imediately.

The lengths of the approximating words: proof of (1.10). For the proof of (1.10) it suffices to show that

$$
l_{j k}^{\prime}=\left|\omega_{j k}^{\prime}\right|=e^{O(k)} \text {. }
$$

This will imply the similar statement for $l_{j k}$. By definition and (2.6) (recall that $1 \leq j \leq k$ ),

$$
l_{j k}^{\prime} \leq n \max _{i} r_{j ; i k} \max _{i}\left|q_{j, i k}\right|,\left|q_{j ; i k}\right|<4^{j}\left(\left|p_{i k}\right|+\left|R_{i}\right|\right)=e^{j \ln 4+O(k)}=e^{O(k)} .
$$

Now for the proof of (6.9) it suffices to prove a similar asymptotics for $r_{j ; i k}$. Indeed, by definition, $r_{j ; i k} \leq \tau_{j ; i k} s_{i k}^{-j}(0), s_{i k}(0)=s_{1 k}(0) \frac{c_{i k}}{c_{1 k}}(0)$,

$$
\tau_{j ; i k}=O\left(\operatorname{dist}\left(x_{j-1, k}, 1\right)=O\left(\operatorname{dist}\left(\omega_{j-1, k}(A, B), x_{k}\right)\right) \leq O\left(\widetilde{V}\left(2 s_{1 k}(0)\right)^{j-1}\right) .\right. \text { Hence, }
$$

$r_{j, i k}=O\left(\widetilde{V} 2^{j} s_{1 k}^{-1}(0)\left(\frac{c_{1 k}}{c_{i k}}(0)\right)^{j}\right)=e^{O(k)}$ (recall that $\left.c_{i k}(0) \rightarrow c_{i}(0)>0\right)$. This together with (6.10) proves inequality (1.10).

The derivatives: proof of (1.11). To show that the derivatives of $\omega_{j k}(a(u), b(u))$ are uniformly bounded in one and the same $\delta$ - neighborhood of 0 , it suffices to show that the derivatives of $\omega_{j k}^{\prime}(a(u), b(u))$ decrease exponentially: there exist $c_{8}, \delta>0$, a $0<s<1$ such that the norm of the derivative of $\omega_{j k}^{\prime}(a(u), b(u))$ is less than $c_{8} s^{j k}$ in $I_{\delta}$, whenever $k$ is large enough and $j \leq k$. By the boundedness of the derivatives of the vector functions $v_{j ; i k}$, for the proof of the previous statement it suffices to prove the same bound for the derivatives of the functions

$$
y_{j ; i k}(u)=r_{j ; i k} s_{i k}^{j}(u):\left\|y_{j, i k}^{\prime}\right\|<c^{\prime} \widetilde{s}^{j k}, \widetilde{s} \text { is independent on } j, k .
$$

As it is shown below, (6.11) is implied by the following 
6.1 Proposition For any $M, c^{\prime}>0,0<s<1$ there exist $c^{\prime \prime}, \delta>0,0<p<1$ satisfying the following statement. Let $0<\sigma(u)<s$ be a $C^{1}$ function defined in a neighborhood of 0 in $\mathbb{R}^{n}$ whose derivative has norm less than $M$. Let $r_{k}$ be a sequence of positive numbers such that

$$
r_{k} \sigma^{k}(0)<c^{\prime} s^{k}
$$

Then the function $\phi_{k}(u)=r_{k} \sigma^{k}(u)$ has derivatives with norm less than $c^{\prime \prime} p^{k}$ in $I_{\delta}$.

Proof By the composition derivative formula, without loss of generality we assume that $\sigma$ is a linear shift:

$$
\sigma(u)=u+u_{0}, \text { thus, } \phi_{k}(u)=r_{k}\left(u+u_{0}\right)^{k} .
$$

By (6.12), $r_{k} u_{0}^{k}<c^{\prime} s^{k}$, hence,

$$
\phi_{k}(u)=\phi(0)\left(\frac{u+u_{0}}{u_{0}}\right)^{k}<c^{\prime}\left(s \frac{u+u_{0}}{u_{0}}\right)^{k} .
$$

Choose a $\delta>0$ such that $s \frac{u_{0}+\delta}{u_{0}}<\frac{s+1}{2}<1$. Then $\phi_{k}(u)<c^{\prime} \widetilde{s}^{k}, \widetilde{s}=\frac{s+1}{2}<1$, whenever $|u|<\delta$. Hence,

$$
\phi_{k}^{\prime}(u)=k \phi_{k}(u)\left(u+u_{0}\right)^{-1}<\left(u_{0}-\delta\right)^{-1} c^{\prime} k \widetilde{s}^{k} .
$$

Take a $p$ such that $\widetilde{s}<p<1$ (say, $p=\frac{\widetilde{s}+1}{2}$ ). Then there exists a $c^{\prime \prime}>0$ such that the latter right-hand side is less than $c^{\prime \prime} p^{k}$ for any $k$. The Proposition is proved.

Let us prove (6.11). By definition, one has

$y_{j ; i k}(0)=r_{j ; i k} s_{i k}^{j}(0)=O\left(\operatorname{dist}\left(x_{j-1, k}, 1\right)\right) \leq O\left(\widetilde{V}\left(2 s_{1 k}(0)\right)^{j-1}\right), s_{1 k}(u)=c_{1 k}(u) s_{1}^{k}(u)=\sigma_{i k}^{k}(u)$,

where the functions $\sigma_{i k}(u)=s_{1}(u)\left(c_{1 k}\right)^{-\frac{1}{k}}(u)$ are uniformly bounded with derivatives (and less than and bounded away from 1 ) on one and the same neighborhood of 0 . Applying Proposition 6.1 to them yields (6.11). The bound (1.11) of the derivatives is proved. Thus, Theorem 1.13 is proved modulo the existence of the words $h_{i}, R_{i}$ and the numbers $r_{i j}$ such that (6.4) and (6.5) hold.

The construction of $r_{i j}$ and the words $h_{i}$ and $R_{i}$. We do our construction of the words $h_{i}$ and the numbers $r_{i j}$ (at the end of the Subsection) so that

$$
p_{i k}(a(u), b(u))=\exp \left(s_{1}^{k}(u) \nu_{i k}(u)\right), \nu_{i k}(u) \in T_{1} G
$$

$$
\nu_{i k}(u) \rightarrow \nu_{i}(u) \text { uniformly with derivatives in some neighborhood of } 0 \text {, as } k \rightarrow \infty,
$$

for each $f_{i}=\nu_{i}(0) \in T_{1} G$ the eigenvector $v_{f_{i}}$ of $a d_{f_{i}}$ is well-defined,

$$
\text { the vectors } v_{f_{i}} \text { form a basis in } T_{1} G \text {. }
$$

The vectors $v_{f_{i}}$ yield the exponential chart (independent on $k$ ) on a neighborhood of unity in $G$ :

$$
\left(t_{1}, \ldots, t_{n}\right) \mapsto \exp \left(t_{1} v_{f_{1}}\right) \ldots \exp \left(t_{n} v_{f_{n}}\right)
$$


This chart contains $w_{k}^{-1}(A, B)$, whenever $k$ is large enough, since, the latter converges to 1 . Let us fix some $n$ words $R_{i}, i=1, \ldots, n$, so that each value $R_{i}(A, B)$ lies in the previous exponential chart close enough to the $t_{i}$ - axis (as will be specified below). Then (6.13)-(6.16) imply (6.4) and (6.5) with $V_{i}(0)=v_{f_{i}}$ (after appropriate normalization of $\left.v_{f_{i}}\right), c_{i k}(u)=$ $s\left(\nu_{i k}(u)\right)$, as in the proof of Lemma 2.4.

Now let us construct the words $h_{i}$. Conditions (6.15) and (6.16) hold for an open set (in the usual topology) of the space of collections $f=\left\{f_{1}, \ldots, f_{n}\right\} \subset T_{1} G$ and are invariant under multiplication of the vectors $f_{i}$ by constants. Let us fix one of the latter collections and denote it $\widetilde{f}=\left\{\widetilde{f}_{1}, \ldots \widetilde{f}_{n}\right\}$.

Let $\widetilde{v}_{1}(u)$ be the vector function from (2.5) corresponding to $w_{1 k}: \widetilde{v}_{1}(u)=\lim _{k \rightarrow \infty} v_{1 k}(u)$, $v_{1}=\widetilde{v}_{1}(0)$. For a generic collection $h_{2}, \ldots, h_{n} \in G$ (we put $h_{1}=1$ ) the vectors

$$
\widetilde{\nu}_{i}=A d_{h_{i}} v_{1}, i=1, \ldots, n,
$$

form a basis in $T_{1} G$. This follows from the irreducibility of the adjoint. We can choose $h_{i}=h_{i}(A, B)$ to be words in $(A, B)$ (density). Let us fix $h_{i}(A, B)$. Then each $\tilde{f}_{i}$ is a linear combination of the $\widetilde{\nu}_{i}^{\prime} \mathrm{s}$ with real coefficients. One can replace the latter combinations by integer ones:

$$
f_{i}=\sum_{j=1}^{n} r_{i j} \widetilde{\nu}_{j}, r_{i j} \in \mathbb{Z}
$$

so that the projectivizations of $f_{i}$ be arbitrarily close to those of $\tilde{f}_{i}$, in particular, so that the previously mentioned conditions (6.15) and (6.16) hold for the new vectors $f_{i}$. Let us fix those $r_{i j}$. Then the corresponding words $p_{i k}$ from (6.2) satisfy (6.13)-(6.16). Indeed, let us prove (6.13). By (2.5), one has

$$
h_{i} w_{1 k} h_{i}^{-1}(a(u), b(u))=\exp \left(s_{1}^{k}(u) \widetilde{v}_{i k}(u)\right), \widetilde{v}_{i k}(u) \rightarrow A d_{h_{i}(a(u), b(u))} \widetilde{v}_{1}(u), \text { as } k \rightarrow \infty,
$$

uniformly on a neighborhood of 0 . Therefore,

$$
p_{i k}(a(u), b(u))=\exp \left(s_{1}^{k}(u) \nu_{i k}(u)\right), \nu_{i k}(u)=\sum_{j=1}^{n} r_{i j} \widetilde{v}_{j k}(u)+o(1), \text { as } k \rightarrow \infty .
$$

This implies (6.13) and (6.14). By construction, $\nu_{i k}$ converge uniformly with derivatives on a neighborhood of 0 , since so do $\widetilde{v}_{j k}$ (say, $\nu_{i k} \rightarrow \nu_{i}$ ), and $\nu_{i}(0)=f_{i}$. Then statements (6.15) and (6.16) follow immediately from construction. The proof of Theorem 1.13 is completed.

I am grateful to É.Ghys who had attracted my attention to the problem. I am grateful to him and also to J.-F.Quint, B.Sevennec for helpful conversations. A significant part of the present proof of the Main Technical Lemma 2.7 was obtained during my stay at RIMS (Kyoto, January 2004). I wish to thank RIMS for hospitality and support.

The research was supported by part by Russian Foundation for Basic Research (RFFI) grants 02-01-00482, 02-01-22002.

\section{References}

[A] Arnold, V.I. Dopolnitelnye glavy teorii obyknovennykh differentsialnykh uravnenii. (Russian) [Supplementary chapters to the theory of ordinary differential equations] - "Nauka", Moscow, 1978. 
[A2] Arnold, V.I. Matematicheskie metody klassicheskoi mekhaniki. (Russian) [Mathematical methods of classical mechanics] Third edition. - "Nauka", Moscow, 1989.

[AGK] Apanasov, B.N., Gusevskii N.A., Krushkal, S.L., Kleinian groups and uniformization in examples and problems (in Russian). - Novosibirsk: Nauka, 1981.

[BG] Breuillard, E., Gelander, T. On dense free subgroups of Lie groups. - J.Algebra, 261 (2003), No. 2, 448-467.

[GJS] Gamburd, A; Jakobson, D; Sarnak, P. Spectra of elements in the group ring of $S U(2)$. - J. Eur. Math. Soc. (JEMS) 1 (1999), no. 1, 51-85.

[CG] Ghys, É., Carrière, Y. Relations d'équivalence moyennables sur les groupes de Lie. - C. R. Acad. Sci. Paris Sér. I Math, vol. 300 (1985), No.19, 677-680.

[E] Epstein, D. B. A. Almost all subgroups of a Lie group are free. - J. Algebra 191971 261-262.

[IP] Ilyashenko, Yu.S.; Pyartli, A.S. The monodromy group at infinity of a generic polynomial vector field on the complex projective plane. - Russian J. Math. Phys. 2 (1994), no. $3,275-315$.

[KR] Kaloshin, V., Rodnianski, I. Diophantine properties of elements of $S O(3)$. - Geom. Funct. Anal. 11 (2001), no. 5, 953-970.

[L] Lyubich, M. Yu. Dynamics of rational transformations: topological picture. (Russian) - Uspekhi Mat. Nauk 41 (1986), no. 4(250), 35-95, 239.

[S] Slodkowski, Z. Holomorphic motions and polynomial hulls. - Proc. Amer. Math. Soc. 111 (1991), no. 2, 347-355.

[ST] Sullivan, D.P.; Thurston, W.P. Extending holomorphic motions. - Acta Math. 157 (1986), no. 3-4, 243-257.

[T] Tits, J. Free subgroups in linear groups. - J.Algebra, vol. 20 (1972), 250-270.

[VO] Vinberg, E. B.; Onishchik, A. L. Seminar po gruppam Li i algebraicheskim gruppam. (Russian) [A seminar on Lie groups and algebraic groups] - Second edition. URSS, Moscow, 1995.

CNRS, Unité de Mathématiques Pures et Appliquées, M.R., École Normale Supérieure de Lyon

46 allée d'Italie, 69364 Lyon Cedex 07 France

Email: aglutsyu@umpa.ens-lyon.fr 